University of Wollongong

Research Online

Faculty of Engineering and Information

Faculty of Engineering and Information

Sciences - Papers: Part B

Sciences

2018

Optimisation of the design of SOI microdosimeters for hadron therapy quality assurance

David Bolst

University of Wollongong, dbolst@uow.edu.au

Susanna Guatelli

University of Wollongong, susanna@uow.edu.au

Linh T. Tran

University of Wollongong, tltran@uow.edu.au

Anatoly B. Rosenfeld

University of Wollongong, anatoly@uow.edu.au

Follow this and additional works at: https://ro.uow.edu.au/eispapers1

Part of the Engineering Commons, and the Science and Technology Studies Commons

Research Online is the open access institutional repository for the University of Wollongong. For further information contact the UOW Library: research-pubs@uow.edu.au 


\title{
Optimisation of the design of SOI microdosimeters for hadron therapy quality assurance
}

\author{
Abstract \\ Silicon-on-insulator (SOI) microdosimeters offer a promising method for routine quality assurance (QA) \\ for hadron therapy due to their ease of operation and high spatial resolution. However, one complication \\ which has been shown previously is that the traditional use of the mean chord length, (ICauchy), calculated \\ using Cauchy's formula, for SOI devices in clinical carbon ion fields is not appropriate due to the strong \\ directionality of the radiation field. In a previous study, we demonstrated that the mean path length, (IPath), \\ which is the mean path of charged particles in the sensitive volume (SV), is a more appropriate method to \\ obtain microdosimetric quantities and biological relevant values, namely the relative biological \\ effectiveness (RBE) by means of the microdosimetric kinetic model. The previous work, which was \\ limited to mono-energetic ${ }^{12} \mathrm{C}$ ion beams typical of heavy ion therapy $(\mathrm{HIT})$, is extended here to \\ investigate the $\left(I_{\text {Path }}\right)$ in a pristine proton beam as well as for spread out Bragg peaks (SOBP) for both \\ proton and carbon ion clinical beams. In addition, the angular dependence of the SOI device for a number \\ of different SV designs is also investigated to quantify the effects which the alignment has on the (IPath). \\ It is demonstrated that the (IPath) can be accurately estimated along the depth of a pristine or SOBP using \\ the energy deposition spectra for both proton and ${ }^{12} \mathrm{C}$ ion beams. This observation allows a quick and \\ accurate estimation of the $\left(I_{P a t h}\right)$ for experimental use. \\ Disciplines \\ Engineering | Science and Technology Studies

\section{Publication Details} \\ Bolst, D., Guatelli, S., Tran, L. T. \& Rosenfeld, A. B. (2018). Optimisation of the design of SOI \\ microdosimeters for hadron therapy quality assurance. Physics In Medicine And Biology, 63 (21), \\ 215007-1-215007-15.
}




\title{
Optimisation of the design of SOI microdosimeters for hadron therapy quality assurance
}

\author{
D. Bolst ${ }^{1}$, S. Guatelli ${ }^{1}$, L. T. Tran ${ }^{1}$ and A. B. Rosenfeld ${ }^{1}$ \\ ${ }^{1}$ Centre for Medical Radiation Physics, University of Wollongong, Australia \\ E-mail: db001@uowmail.edu.au
}

\begin{abstract}
Silicon-on-insulator (SOI) microdosimeters offer a promising method for routine quality assurance (QA) for hadron therapy due to their ease of operation and high spatial resolution. However, one complication which has been shown previously is that the traditional use of the mean chord length, $\left\langle l_{\text {Cauchy }}\right\rangle$, calculated using Cauchy's formula, for SOI devices in clinical carbon ion fields is not appropriate due to the strong directionality of the radiation field. In a previous study, we demonstrated that the mean path length, $\left\langle l_{\text {Path }}\right\rangle$, which is the mean path of charged particles in the sensitive volume (SV), is a more appropriate method to obtain microdosimetric quantities and biological relevant values, namely the relative biological effectiveness (RBE) by means of the microdosimetric kinetic model. The previous work, which was limited to mono-energetic ${ }^{12} \mathrm{C}$ ion beams typical of heavy ion therapy (HIT), is extended here to investigate the $\left\langle l_{P a t h}\right\rangle$ in a pristine proton beam as well as for spread out Bragg peaks (SOBP) for both proton and carbon ion clinical beams. In addition, the angular dependence of the SOI device for a number of different SV designs is also investigated to quantify the effects which the alignment has on the $\left\langle l_{P a t h}\right\rangle$. It is demonstrated that the $\left\langle l_{\text {Path }}\right\rangle$ can be accurately estimated along the depth of a pristine or SOBP using the energy deposition spectra for both proton and ${ }^{12} \mathrm{C}$ ion beams. This observation allows a quick and accurate estimation of the $\left\langle l_{\text {Path }}\right\rangle$ for experimental use.
\end{abstract}

Keywords: Microdosimetry, Hadron therapy, Silicon-on-insulator (SOI), Geant4

\section{Introduction}

Silicon-on-insulator (SOI) microdosimeters are convenient detectors for quality assurance (QA) in hadron therapy thanks to their small and portable design and simple operation compared to traditional tissue equivalent proportional counters (TEPC) which are not well suited for QA. The advantage of using microdosimeters for QA in hadron therapy, especially ${ }^{12} \mathrm{C}$ ion beams, is that they provide information that may be used to estimate the beam's relative biological effectiveness (RBE) for local tumour control. One way which the RBE may be estimated from microdosimetric spectra is by using a modified microdosimetric kinetic model (MKM) which is usually calculated for an endpoint of $10 \%$ cell survival survival, [1] [2]. The modified MKM makes changes from the original formulated by Hawkins [3] [4].

Current SOI microdosimeters designed at the Centre for Medical Radiation Physics (CMRP), University of Wollongong, feature fully etched sensitive volumes (SV) which have excellent charge collection efficiency and yields. 
There are two currently available fully etched SOI detectors designed by the CMRP, called "Bridge" and "Mushroom". Both designs are made of arrays of SVs connected together with aluminium contacts and connected in alternating channels. The Bridge's arrays are made up of well-defined three dimensional (3D) silicon parallelepiped SVs connected together by smaller sized silicon bridging volumes while the Mushroom's SVs are cylindrical volumes. Details on the two alternative designs are provided in [5] and [6]. Both devices are radiation hard, the Bridge is an n-type silicon detector with a resistivity of $3 \mathrm{k} \Omega \mathrm{cm}$ and the Mushroom is a p-type with a higher resistivity of $10 \mathrm{k} \Omega \mathrm{cm}$. The devices have been successfully tested in a range of different radiation environments including in-field [7] and out-of-field [8] measurements in scanning proton beams, passive ${ }^{12} \mathrm{C},{ }^{14} \mathrm{~N},{ }^{16} \mathrm{O}$ and scanning ${ }^{12} \mathrm{C}$ ion beams [9]. A low energy ${ }^{12} \mathrm{C}$ beam with a total energy of $70 \mathrm{MeV}$ corresponding to a range of $\sim 180 \mu \mathrm{m}$ in water has also been characterised with the SOI devices. This final beam represents a particularly high LET field due to the limited range straggling causing the beam to stop in a narrow range of a few $\mu \mathrm{m}[5]$.

Microdosimetry involves measuring the energy deposition, $E$, in micron-sized volumes and converting the $E$ to lineal energy, $y[10]$. The lineal energy is traditionally obtained by dividing $E$ by the mean chord length, $\langle l\rangle$, as shown in equation 1 , where the $\langle l\rangle$ is calculated using Cauchy's equation [11] as shown in equation 2, where $V$ and $S$ are the volume and the surface area of the SV of interest, respectively. One notable complication with using SOI microdosimeters compared to spherical TEPCs is that the radiation fields typical of hadron therapy are highly directional. Thus, the conventional method of using Cauchy's formula to calculate the mean chord length $\langle l\rangle$ can be inappropriate. This happens because Cauchy's formula is for isotropic or $\mu$-randomness fields [12] where the distribution of particles traversing the SV is uniform over all angles, while for hadron therapy the radiation field is not isotropic, with particles traversing from one dominant direction at a time.

$$
\begin{gathered}
y=\frac{E}{\langle l\rangle}, \\
\left\langle l_{\text {Cauchy }}\right\rangle=\frac{4 V}{S}
\end{gathered}
$$

Previous work described in [13] investigated the use of the mean path length, $\left\langle l_{\text {Path }}\right\rangle$, as an alternative to the $\left\langle l_{\text {Cauchy }}\right\rangle$ in ${ }^{12} \mathrm{C}$ ion therapy using Geant4 Monte Carlo simulations [14] [15] [16]. The $\left\langle l_{\text {Path }}\right\rangle$ is the mean path length of charged particles which traverse the SV as opposed to the random distributions of chords within the volume used to calculate $\left\langle l_{\text {Cauchy }}\right\rangle$.

In [13] the $\left\langle l_{\text {Path }}\right\rangle$ was calculated for a microdosimeter placed at various positions along the Bragg curve of a mono-energetic ${ }^{12} \mathrm{C}$ ion beam with clinical energy within a water phantom. The study showed that the $\left\langle l_{\text {Cauchy }}\right\rangle$ was inappropriate for the strong directionality of the radiation field and instead the $\left\langle l_{\text {Path }}\right\rangle$ is preferable. Therefore the lineal energy is calculated more precisely with equation 3 :

$$
y=\frac{E}{\left\langle l_{\text {Path }}\right\rangle}
$$

Furthermore, it was found that the thickness of the SV of the SOI microdosimeter provided a good approximation for the $\left\langle l_{P a t h}\right\rangle$ calculated in-field when estimating the RBE using the MKM.

This study extends the previous work which was limited to investigating the $\left\langle l_{\text {Path }}\right\rangle$ for monoenergetic ${ }^{12} \mathrm{C}$ ion beams. Here the $\left\langle l_{\text {Path }}\right\rangle$ is examined for the case of a mono-energetic proton beam and spread out Bragg peaks (SOBP) for both incident protons and ${ }^{12} \mathrm{C}$ ions.

This work continues to further investigate the silicon-based experimental microdosimetric approach, in order to determine more accurately important quantities for treatment planning such as the dose equivalent and the RBE. It also supports the further optimisation of the SV's 
design, particularly in terms of its dimensions, for the application of SOI microdosimeters in hadron therapy QA.

When irradiated in hadron therapy beams, the SOI microdosimeters are usually set face on to the incident beam. Nevertheless, misalignments of few degrees may happen. Thus, the angular dependence of the $\left\langle l_{\text {Path }}\right\rangle$ is investigated to quantify the effect of such possible experimental misalignment on the $\left\langle l_{\text {Path }}\right\rangle$ and to see the general effect of different detector alignments with the beam.

In addition, this work investigates the effect on the $\left\langle l_{\text {Path }}\right\rangle$ value given by different diameters (10 $\mu \mathrm{m}$ and larger) of the cylindrical Mushroom SV while keeping the SV thickness in the direction of beam incidence constant and equal to $10 \mu \mathrm{m}$. Such thickness is adopted as currently it is the most common SV thickness of fabricated SOI microdosimeters.

\section{Materials and Methods}

In this study Geant4 (version 10.2p1) was adopted as Monte Carlo code to investigate the $\left\langle l_{\text {Path }}\right\rangle$ in proton and ${ }^{12} \mathrm{C}$ ion therapies. First the mean geometrical path length in the SV, $\left\langle l_{G e o}\right\rangle$, was calculated. This approach allowed the investigation of the effect on the $\left\langle l_{\text {Path }}\right\rangle$ produced by different alignments of the detector with the incident beam direction and by the angular spread or divergence of the particles incident on the SV, based on geometrical considerations only (neglecting the effect of the physical interactions). The difference between the $\left\langle l_{G e o}\right\rangle$ and the $\left\langle l_{\text {Path }}\right\rangle$ is that the $\left\langle l_{\text {Path }}\right\rangle$ considers all physical processes and particles created while $\left\langle l_{\text {Geo }}\right\rangle$ only considers different defined distributions of geometrical tracks traversing the SV. The advantage of studying the $\left\langle l_{G e o}\right\rangle$ is that it allows for the trend of the $\left\langle l_{P a t h}\right\rangle$ to be studied for general cases of alignment and divergence of the beam passing the SV while the $\left\langle l_{\text {Path }}\right\rangle$ represents a complicated mix of particles which have angular distributions that often vary with position in the phantom because of their physical interactions.

The study of the $\left\langle l_{G e o}\right\rangle$ was followed by the calculation of the mean path length in the SV modelled with a vacuum material, $\left\langle l_{\text {Path }}\right\rangle$, following the same methodology described in [13], where the SV material is modelled as a vacuum to remove any physical interactions and the distance a charged particle traverses the SV is recorded. The study has been done for both mono-energetic and clinical SOBP proton and ${ }^{12} \mathrm{C}$ ion beams using different SV diameter sizes.

\subsection{Calculation of the mean geometrical path length}

For calculations of the $\left\langle l_{G e o}\right\rangle$ a geantino beam was simulated. In Geant4 geantinos are particles which do not have any physical interactions. Such particles are well suited for geometrical studies. For both configuration studies, a $1 \mathrm{~mm}$ diameter beam was generated $0.01 \mu \mathrm{m}$ from the top of the SV, independently from the SV's alignment angle (shown in figure 1 (a) and (b)).

For the case representing the angular alignment of the detector with the beam, a parallel beam was simulated incident upon a single SV placed at angles varying between $0^{\circ}$ and $90^{\circ}$ with respect to the beam as shown in figure 1 (a). $0^{\circ}$ and $90^{\circ}$ correspond to face on (with the SV perfectly aligned with the beam) and edge on configurations, respectively. For context, the accuracy of the positioning of the CMRP detector and phantom system with the beam is within $1^{\circ}$ but larger angles are investigated for the interest of less precise setups and for general considerations. The simulated parallel beam differs slightly from real world therapeutic beams which have divergence, often a few milliradians at iso-centre, due to the shaping of the beam.

The second configuration, shown in figure 1 (b), investigates the effect of the angular distribution or divergence of the incident beam. In this case the $\left\langle l_{G e o}\right\rangle$ was calculated for different SV diameters when irradiated by a cone beam, varying the aperture angle $\theta$ as shown in figure 1 (b). The particles are generated with a random direction within the cone. In this case the incident beam originates from the same $1 \mathrm{~mm}$ diameter used in the alignment configuration to ensure the entire area of the SV is equally irradiated. 
For both configurations the thickness of the SV is fixed to $10 \mu \mathrm{m}$ and the geometrical path length is studied by varying the diameter of the SV. The path length in the SV was calculated as the distance between the point of entrance and exit of the geantino in the SV. The $\left\langle l_{G e o}\right\rangle$ was calculated as the average value of the geometrical path length distribution.

\subsection{Calculation of $\left\langle l_{\text {Path }}\right\rangle$ in radiation fields of interest for hadron therapy}

Figure 1 (c) shows the simulation set-up for calculating the $\left\langle l_{\text {Path }}\right\rangle$ in different beams, both monoenergetic and SOBP beams were simulated for proton and ${ }^{12} \mathrm{C}$ ion beams. The mono-energetic beams were $150 \mathrm{MeV}$ and $290 \mathrm{MeV} / \mathrm{u}$ for proton and ${ }^{12} \mathrm{C}$ ions, respectively, which correspond to a range of $\sim 160 \mathrm{~mm}$ in water. For the simulated proton beams an energy sigma of $1 \%$ was applied while for the ${ }^{12} \mathrm{C}$ ion beams a $0.2 \%$ sigma was used. The SOBPs were produced using multiple energy weights. The proton SOBP was weighted to deliver a uniform physical dose 50 $\mathrm{mm}$ in width while the ${ }^{12} \mathrm{C}$ ion SOBP was weighted to give a uniform biological dose $60 \mathrm{~mm}$ in width. The incident beam position was modelled as a Gaussian with a $\sigma$ of $11 \mathrm{~mm}$. The beam was incident upon a $30 \times 30 \times 30 \mathrm{~cm}^{3}$ water phantom with $40 \mathrm{~cm}$ of air between the beam's starting position and the surface of the phantom.

The Mushroom microdosimeter was positioned at various depths along the Bragg curve/spread out Bragg peak in water. It was modelled following the same approach of our previous paper [13]. The detector features alternating even and odd channels of SVs embedded in PMMA supported by a $1 \mu \mathrm{m}$ thick $\mathrm{SiO}_{2}$ substrate. The distance between the outside edges of the SVs, is $40 \mu \mathrm{m}$. A zoomed in region of the detector with $10 \mu \mathrm{m}$ diameter SVs is shown in figure 1 (d). The thickness of the SVs along the beam direction was fixed to $10 \mu \mathrm{m}$. The different detector designs investigated included diameters of 10, 20,30, 40 and $50 \mu \mathrm{m}$, alternatively. For larger diameter designs the distance between the SV edges and area that the SVs occupied was kept the same as the $10 \mu \mathrm{m}$ design except that fewer total SVs were placed, with the active area populated with SVs being $2.5 \times 2.5 \mathrm{~mm}^{2}$.

The electromagnetic interactions were modelled using G4 Standard EM option 3. G4HadronPhysicsQGSP_BIC_HP and G4HadronElasticPhysicsHP were used to describe the inelastic and elastic scattering of hadrons, respectively. The G4IonBinaryCascadePhysics was used to describe hadronic interactions of ions, the accuracy of this model was recently compared against experimental fragmentation measurements of a ${ }^{12} \mathrm{C}$ ion beam in water and was found to perform adequately [17]. For neutron interactions up to $20 \mathrm{MeV}$ the neutron High Precision (HP) model was used.

The G4CutsPerRegion allows different thresholds of production of secondary particles in different geometrical components. This functionality was adopted in the simulation to reduce the execution times without affecting the accuracy of the results. In particular the $\delta$-electrons generated by the incident hadron beam are modelled only close to the microdosimeter, in a region surrounding the detector, as shown in figure 1 (c). The cut region has a thickness twice the maximum range of electrons plus the total thickness of the detector $(10 \mu \mathrm{m}$ SV thickness and $1 \mu \mathrm{m}$ substrate), the width of the cut region is the active area of the detector $(2.5 \mathrm{~mm})$ plus twice the maximum electron range. The maximum range of the electrons is approximately $1 \mathrm{~mm}$ and $2.3 \mathrm{~mm}$ for the incident protons and ${ }^{12} \mathrm{C}$ ions, respectively. The total thickness of the cut region is then $2.011 \mathrm{~mm}(2 \times 1 \mathrm{~mm}+10 \mu \mathrm{m}+1 \mu \mathrm{m})$ and $4.611 \mathrm{~mm}$ for the proton and ${ }^{12} \mathrm{C}$ ion beams, respectively, while the total width is $4.5 \mathrm{~mm}$ and $7.1 \mathrm{~mm}$ for the proton and ${ }^{12} \mathrm{C}$ ion beams, respectively. The cut is fixed to $1 \mathrm{~mm}$ and $2.3 \mathrm{~mm}$ outside of the cut region for the proton and ${ }^{12} \mathrm{C}$ incident beam, respectively. In this region the $\delta$ electrons are not modelled because they will never reach the detector and therefore they do not contribute to the microdosimetric spectra. 
(a)

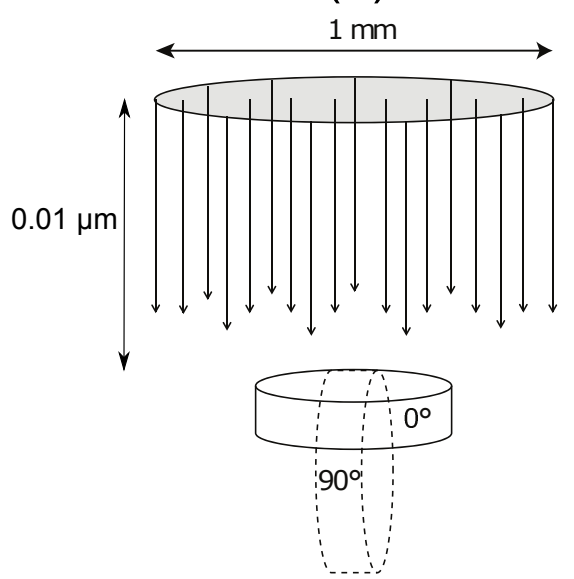

(c)

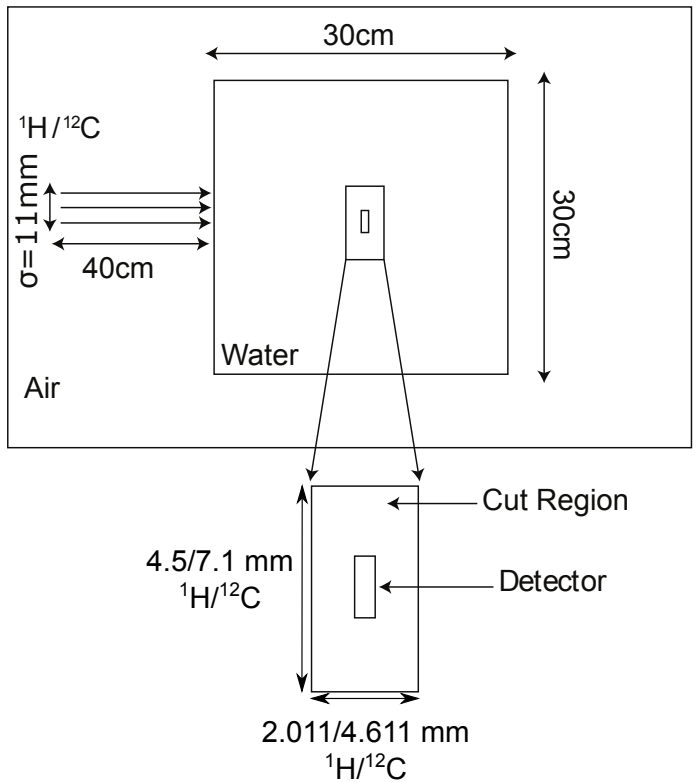

(b)

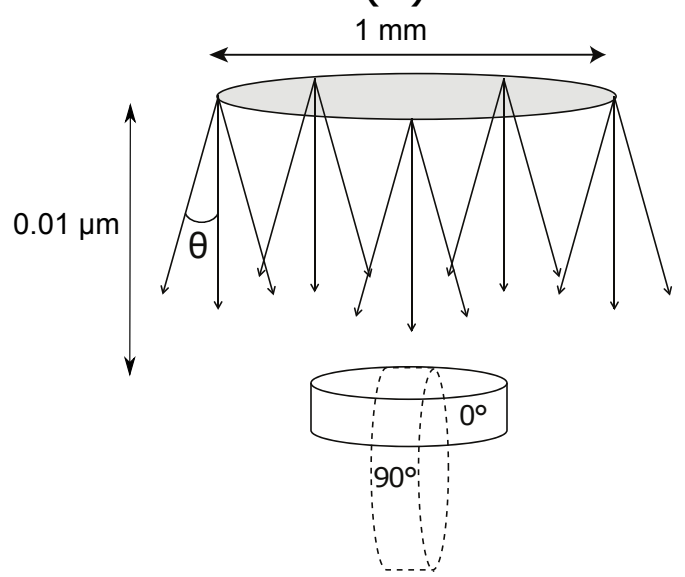

(d)

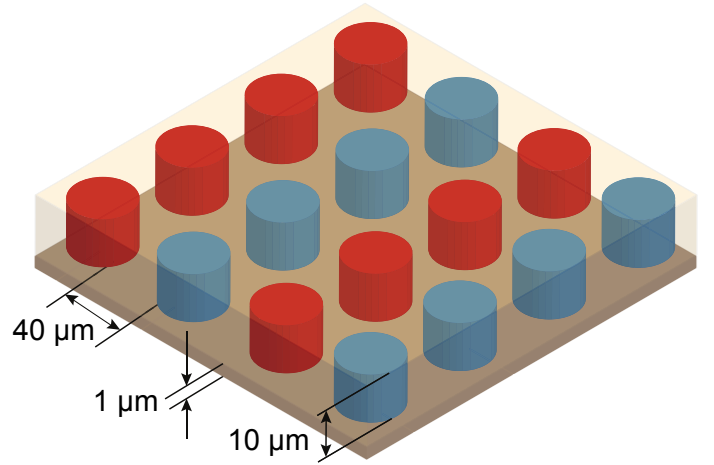

Figure 1: Simulation setups used in this study. (a) and (b) show the simulation setup adopted to study the effect on the $\left\langle l_{G e o}\right\rangle$. (a) studies the effect of the alignment of the SV with the beam direction, the SV is irradiated with a parallel geantino beam. (b) is the setup used to study the effect of the angular spread of the incident geantino beam. (c) depicts the simulation setup used to calculate the $\left\langle l_{\text {Path }}\right\rangle$ in the radiation fields of interest and $(\mathrm{d})$ shows a region of the SOI microdosimeter modelled in the Geant 4 simulation to calculate the $\left\langle l_{\text {Path }}\right\rangle$. All the investigated designs for the $\left\langle l_{\text {Path }}\right\rangle$ had the same thickness of $10 \mu \mathrm{m}$ in the direction of the incident beam and the same distance between the outside of the SVs of $40 \mu \mathrm{m}$. Microdosimeter designs with larger diameter SVs have less SVs in total. The blue and red SVs represent SVs connected together on different channels. 


\subsection{Estimating the $\left\langle l_{\text {Path }}\right\rangle$ using the energy deposition spectra}

The final study involved using the same simulation set-up depicted in figure 1 (c) but replacing the vacuum SV with silicon to calculate the energy deposition. Monte Carlo simulations are a valid tool to determine the $\left\langle l_{\text {Path }}\right\rangle$ when the beam line is well known and accurately described in the simulation. However, in experimental studies the incident radiation field on the microdosimeter by a beam line may not be well known, producing possible inaccuracies between the true and the Monte Carlo-based calculation of the $\left\langle l_{P a t h}\right\rangle$. The effect of the discrepancy between the actual and calculated $\left\langle l_{P a t h}\right\rangle$ values may be further amplified if the design of the SV causes a large variation of the $\left\langle l_{\text {Path }}\right\rangle$ along the Bragg curve leading to the SV thickness being inappropriate as an approximation to the $\left\langle l_{\text {Path }}\right\rangle$.

In order to limit possible inaccuracies in the value of the $\left\langle l_{P a t h}\right\rangle$, deriving from situations as the ones mentioned above, it is convenient to be able to estimate the $\left\langle l_{\text {Path }}\right\rangle$ directly from the response of the detector. [13] demonstrated that there is a unique relationship between the energy deposited in the SV by a mono-energetic ${ }^{12} \mathrm{C}$ ion beam and the $\left\langle l_{\text {Path }}\right\rangle$ at any depth in the phantom. This allows the $\left\langle l_{\text {Path }}\right\rangle$ from experimental measurements to be estimated. In [13] this method was demonstrated for mono-energetic ${ }^{12} \mathrm{C}$ ion beams only. Here we investigate the applicability of the same method to mono-energetic proton and ${ }^{12} \mathrm{C}$ ion beams with energy of $150 \mathrm{MeV}$ and $290 \mathrm{MeV} / \mathrm{u}$, respectively, and clinical proton and ${ }^{12} \mathrm{C}$ ion SOBP beams. Here a $10 \mu \mathrm{m}$ diameter SV was adopted as this design was found to correspond to the worst case of variation of the $\left\langle l_{\text {Path }}\right\rangle$.

With the SV now being silicon the energy deposition spectra were recorded in the silicon SVs and to make the spectrum biologically relevant they were converted to striated muscle by using the conversion factors calculated in [13] of 0.58 and 0.57 for proton and ${ }^{12} \mathrm{C}$, respectively, (note that the value for protons was not published but was done using the same methodology as ${ }^{12} \mathrm{C}$ ions, also the value for protons differs from the value published in [18] which was calculated for water). The conversion factor, $\kappa$, is applied when converting the energy deposited, $E$, spectrum to the lineal energy spectrum, $y$. Instead of using the $\left\langle l_{\text {Path }}\right\rangle$ of the actual silicon SV the $\left\langle l_{\text {Path }}\right\rangle$ of the equivalent muscle volume is obtained by dividing the $\left\langle l_{\text {Path }}\right\rangle$ of silicon by the conversion factor $\kappa,\left\langle l_{\text {Path }, \text { Muscle }}\right\rangle=\left\langle l_{\text {Path }, S i}\right\rangle / \kappa$, so the final corrected lineal energy is $y=\kappa E /\left\langle l_{\text {Path,Si}}\right\rangle$. Once the lineal energy spectra were converted to muscle, the mean of the incident beam's energy deposition peak was retrieved and associated to the calculated $\left\langle l_{\text {Path }}\right\rangle$ at that depth.

Microdosimetric spectra have been calculated in this work for proton and ${ }^{12} \mathrm{C}$ ion beams. The $y d(y)$ distribution on a semi-log plot is the most common way of representing microdosimetric spectra and is equivalent to the dose distribution, $d(y)$, on a linear scale where $d(y)=$ $y f(y) / \int y f(y) d y$ and $f(y)$ is the lineal energy frequency distribution. $y d(y)$ is obtained by rebinning the constant width bins of the $d(y)$ distribution bins into log bins. A detailed description of this method can be found in [19] and Appendix B of the ICRU 36 report [20]. The area for a certain lineal energy bin of the $y d(y)$ (or $d(y)$ ) curve is proportional to the amount of dose deposited in the SV at the particular lineal energy.

\section{Results}

\subsection{Study of the mean geometrical path length.}

Figure 2 shows the calculated $\left\langle l_{G e o}\right\rangle$ when considering different tilt angles and diameters of SVs. The geantinos are incident normally to the SV when aligned at $0^{\circ}$. In general it can be observed that the $\left\langle l_{G e o}\right\rangle$ decreases to a minimum, whose value depends on the specific SV diameter, and then it increases with the tilt angle. The $\left\langle l_{G e o}\right\rangle$ can differ significantly from the $\left\langle l_{\text {Cauchy }}\right\rangle$, reported in table 1 for all the SV diameters considered. When the SV is aligned at smaller tilt angles (less than $\sim 30^{\circ}$ ), it can be observed that the $\left\langle l_{G e o}\right\rangle$ varies much less from its initial value at $0^{\circ}$ for larger diameters. Compared to the $\left\langle l_{G e o}\right\rangle$ at $0^{\circ}$, the value at $15^{\circ}$ for the 10 $\mu \mathrm{m}$ diameter SV has a reduction of approximately $\sim 20 \%$, while for the $20 \mu \mathrm{m}$ diameter SV the 
reduction is $\sim 10 \%$. However, at larger tilt angles the larger the diameter of the SV the more rapidly their value differs from the $0^{\circ}$ value.

\begin{tabular}{c|c}
\hline Diameter $(\mu \mathrm{m})$ & $\left\langle l_{\text {Cauchy }}\right\rangle(\mu \mathrm{m})$ \\
\hline 10 & 6.67 \\
20 & 10 \\
30 & 12 \\
50 & 14.29 \\
100 & 16.67 \\
\hline
\end{tabular}

Table 1: $\left\langle l_{\text {Cauchy }}\right\rangle$ calculated for $10 \mu \mathrm{m}$ thick SVs, with varying diameter.

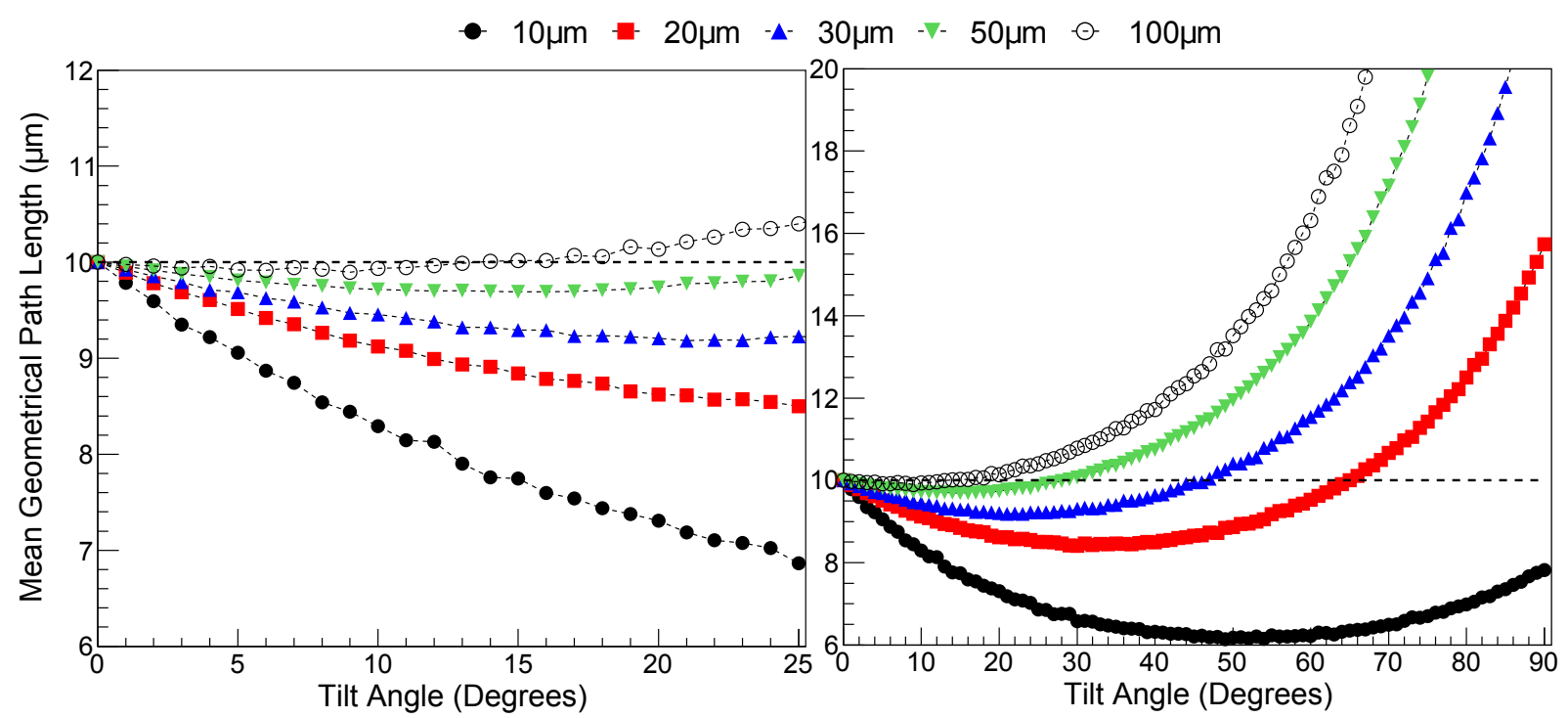

Figure 2: The $\left\langle l_{G e o}\right\rangle$ is plotted against the tilt angle, when varying the SV diameter (reported in the legend). The left plot shows a zoomed in range of the right plot.

Figure 3 shows the effect of the angular spread or divergence of the incident beam, represented in terms of $\theta$ (see figure 1, (b)), when varying the diameter of the SV. The $\left\langle l_{G e o}\right\rangle$ is done for a number of different tilt angles between $0^{\circ}$ and $25^{\circ}$. For smaller $\theta$ (up to $\sim 40^{\circ}$ ), the $\left\langle l_{G e o}\right\rangle$ varies the most for smaller diameter SVs, with the $10 \mu \mathrm{m}$ diameter SV varying between $\sim 7$ and 10 $\mu \mathrm{m}$. In contrast, for large $\theta$ the larger diameter SVs have values of $\left\langle l_{G e o}\right\rangle$ which extend a greater range of values compared to smaller SVs. Similar to the misalignment case, each SV is seen to reach a minimum $\left\langle l_{G e o}\right\rangle$ value depending on the specific diameter of the SV and then it increases again.

When changing the alignment of the detector by altering the tilt angle the variation of the $\left\langle l_{G e o}\right\rangle$ can be seen to reduce, with the reduction being the greatest for the smaller diameters. At a tilt angle of $25^{\circ}$ the $10 \mu \mathrm{m}$ diameter can be seen to provide an almost flat response of the $\left\langle l_{G e o}\right\rangle$ between beam divergences of $0^{\circ}$ and $90^{\circ}$. For the $20 \mu \mathrm{m}$ diameter SV the tilt angle to obtain a flat response is reduced to $\sim 15^{\circ}$, however, the range of the flat response is reduced to a maximum of $\sim 50^{\circ}$. The larger diameter SVs do not experience a strong flat response when adjusting the tilt angle, with the $\left\langle l_{G e o}\right\rangle$ generally increasing with larger beam divergence. 

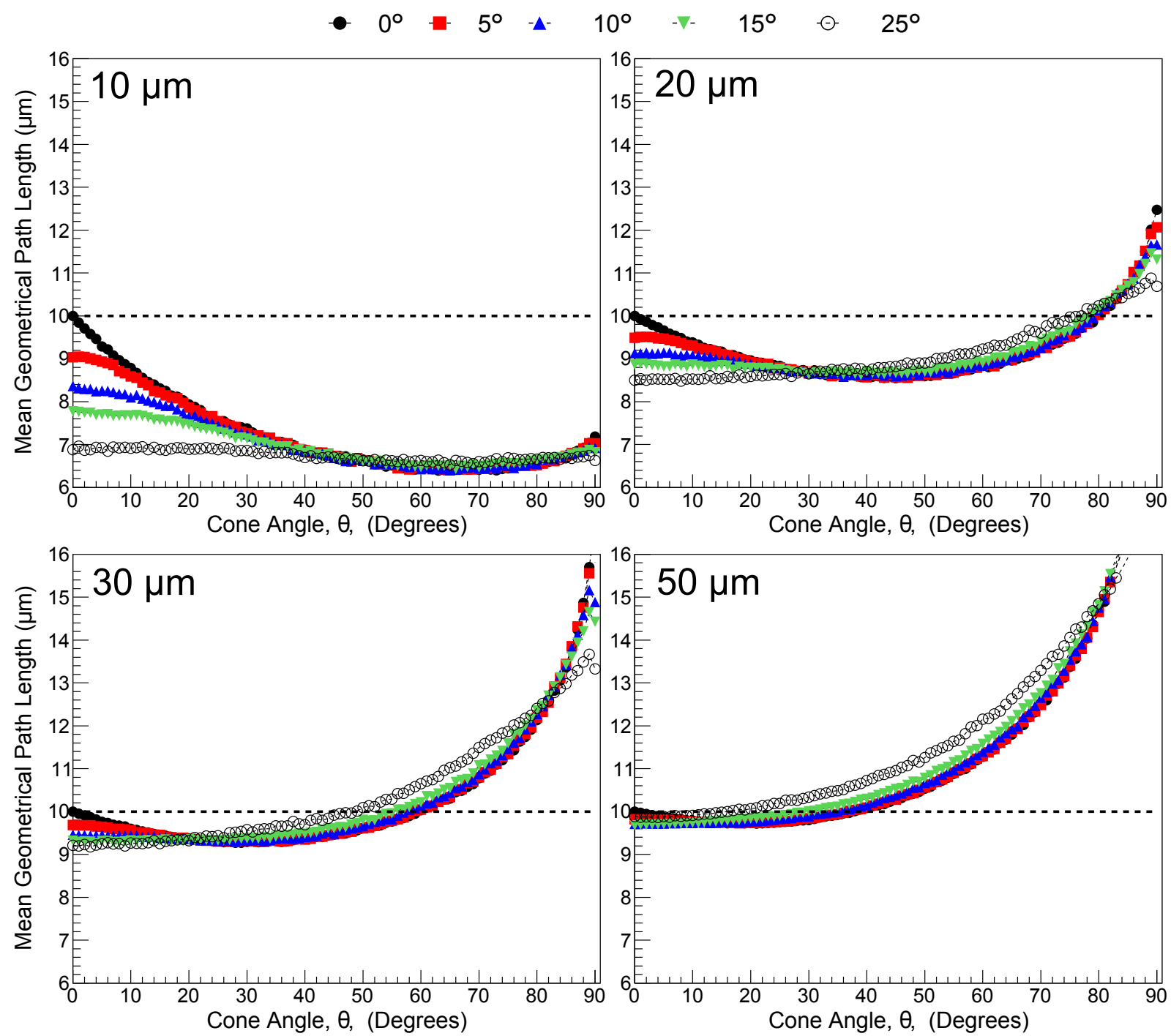

Figure 3: $\left\langle l_{G e o}\right\rangle$ of SVs with a diameter of: $10 \mu \mathrm{m}$ (top left), $20 \mu \mathrm{m}$ (top right), $30 \mu \mathrm{m}$ (bottom left) and $50 \mu \mathrm{m}$ (bottom right). $\left\langle l_{G e o}\right\rangle$ is calculated against the angle $\theta$ (see figure 1 (b)). The tilt angle of detector alignment is shown in the top legend.

\subsection{Characterisation of the Proton and ${ }^{12} \mathrm{C}$ ion Radiation Fields}

The fluence of the incident and secondary particles along the depth of the water phantom in the mono-energetic proton and ${ }^{12} \mathrm{C}$ ion radiation fields under investigation are shown in figure 4 (a) and (b). It can be seen that for the proton beam the fluence of neutrons and protons is fairly constant before the Bragg peak, after which the fluence of secondary protons decreases rapidly while the neutrons are attenuated more slowly. Comparing the fluence of the primary proton and ${ }^{12} \mathrm{C}$ ion beams it can be seen that the ${ }^{12} \mathrm{C}$ ion beam undergoes a much higher amount of attenuation $(\sim 50 \%)$ compared to the proton beam $(\sim 20 \%)$ when reaching the Brag Peak (BP). This is due to the significantly higher nuclear cross-sections of ${ }^{12} \mathrm{C}$ compared to proton beams, which translates to $\sim 4$ orders of magnitude higher fluence of fragments in the ${ }^{12} \mathrm{C}$ ion beam case. Due to the different dominant processes in the two fields the proton beam is dominated by neutrons with a large angular distribution while the ${ }^{12} \mathrm{C}$ ion radiation field is more dominated by more forward directed fragments. 
(a)

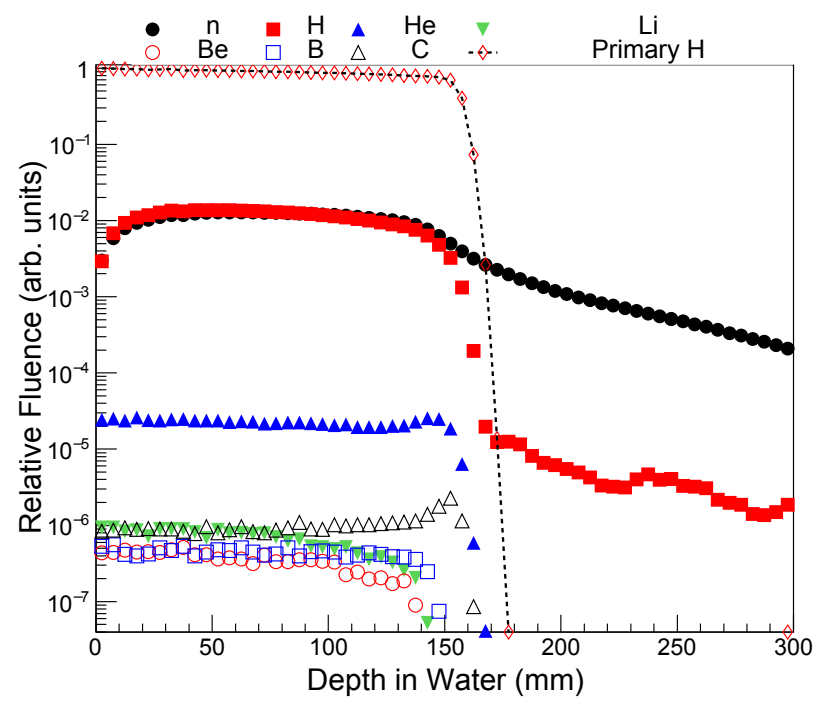

(c)

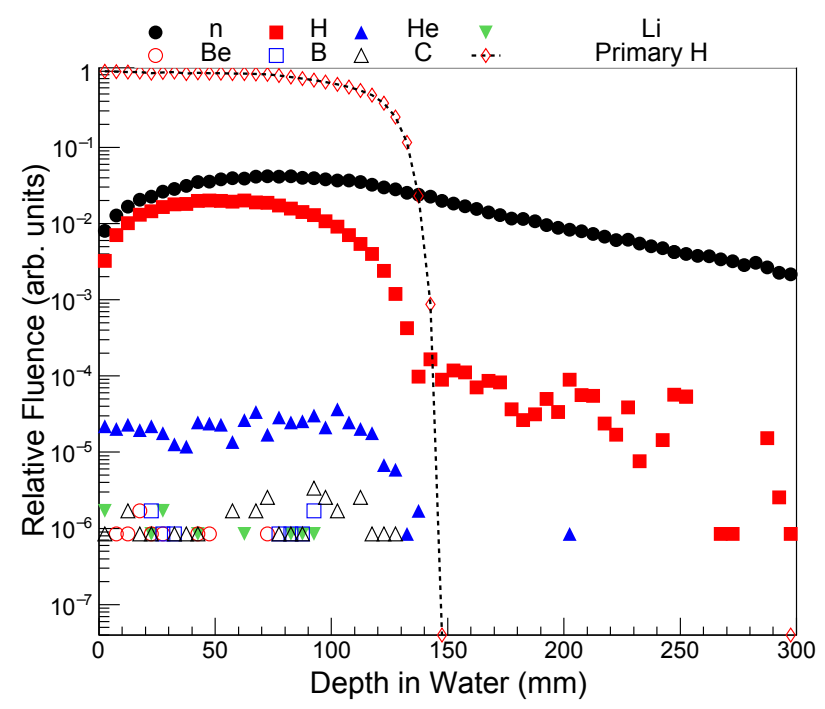

(b)

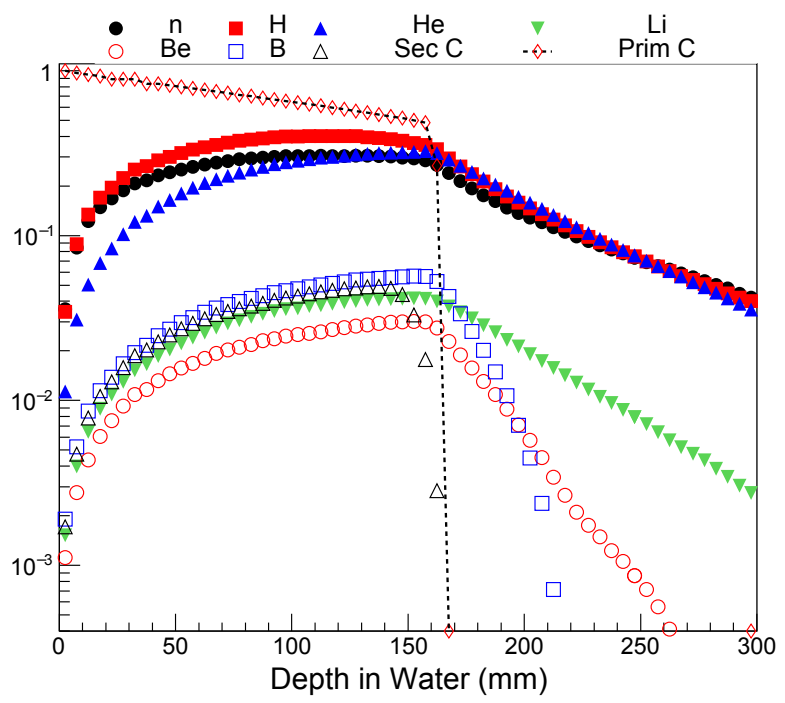

(d)

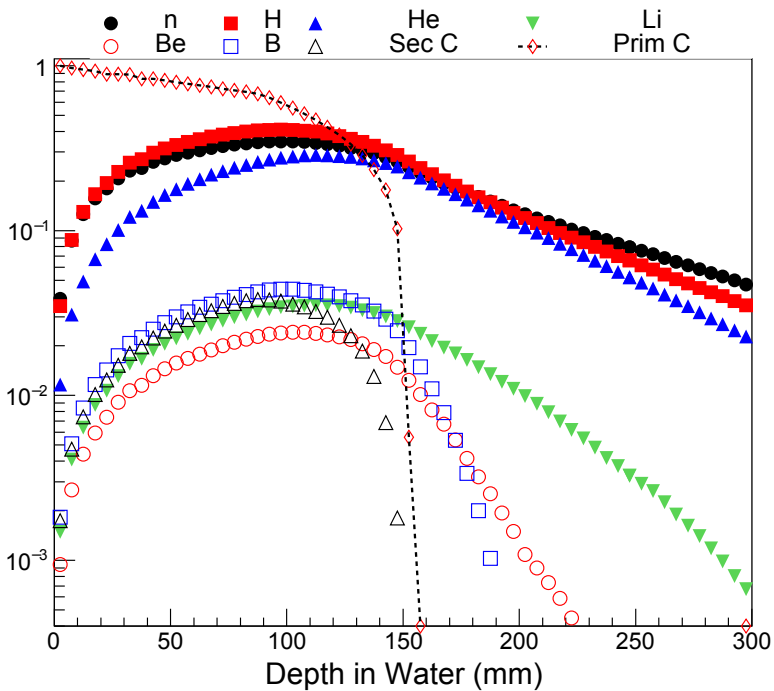

Figure 4: The relative fluence of different nuclear species along the depth of the water phantom for an incident: $150 \mathrm{MeV}$ proton beam (a), a $290 \mathrm{MeV} / \mathrm{u}{ }^{12} \mathrm{C}$ ion beam (b), a proton SOBP (c) and a ${ }^{12} \mathrm{C}$ ion SOBP (d). Note that the minimum of the proton y-axis is three orders of magnitude lower than the ${ }^{12} \mathrm{C}$ axis.

Figure 4 (c) and (d) shows the fluence of secondary particles along the depth in the phantom in the proton and ${ }^{12} \mathrm{C}$ ion SOBP fields. The fluences are higher than in the case of the corresponding mono-energetic beams due to the distal radiation fields from different beam energies accumulating as they stop in the phantom.

Typical proton and ${ }^{12} \mathrm{C}$ ion microdosimetric $y d(y)$ spectra are shown in figure 5 for a 150 $\mathrm{MeV}$ proton and $290 \mathrm{MeV} / \mathrm{u}{ }^{12} \mathrm{C}$ ion beam both placed at a depth of $150 \mathrm{~mm}$ (with an angle of $0^{\circ}$ with the beam, corresponding to the face on configuration). This depth corresponds to the $\mathrm{BP}$ for the proton beam and just before the $\mathrm{BP}$ for the ${ }^{12} \mathrm{C}$ ion beam. The spectra have been converted from silicon (using the $20 \mu \mathrm{m}$ diameter Mushroom SV design) to striated muscle using the conversion factor of 0.58 and 0.57 found previously in [13]. Each plot shows the contribution 
that different particle types make to the total dose deposited in the detector. It can be seen that in the case of the proton beam different particle types have a similar lineal energy while the microdosimetric spectra of the ${ }^{12} \mathrm{C}$ ion beam have a well defined peak due to the primary ${ }^{12} \mathrm{C}$ ions and a lower lineal energy peak due to secondary fragments and electrons. In particular, in the case of proton therapy the lineal energy spectra of the primary and secondary protons overlap along the Bragg curve, because of this it is important to have similar $\left\langle l_{\text {Path }}\right\rangle$ for both the primary beam and secondary ions.
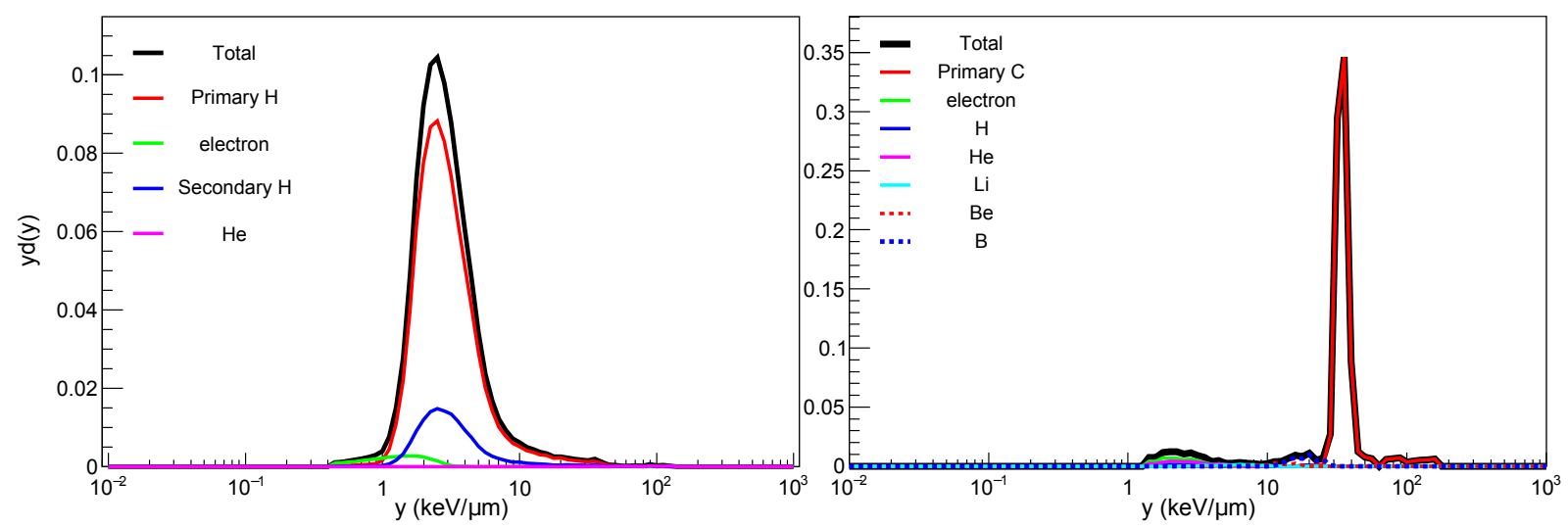

Figure 5: $y d(y)$ spectra of the detector for a $150 \mathrm{MeV}$ proton beam (left) and a $290 \mathrm{MeV} / \mathrm{u}{ }^{12} \mathrm{C}$ ion beam (right), both at $150 \mathrm{~mm}$ in water.

\section{3. $\left\langle l_{\text {Path }}\right\rangle$ in mono-energetic beam}

The mean path length, $\left\langle l_{\text {Path }}\right\rangle$, was calculated for SVs with various diameters, when irradiated by a $150 \mathrm{MeV}$ and $290 \mathrm{MeV} / \mathrm{u}$ pristine proton and ${ }^{12} \mathrm{C}$ ion beam, respectively. The $\left\langle l_{\text {Path }}\right\rangle$ is presented for various depths in the radiation fields as well as tilt angles between the microdosimeter and the incident beam for diameter sizes of 10 and $20 \mu \mathrm{m}$ and are summarised along with 30,40 and $50 \mu \mathrm{m}$ diameters at the end of this section in tables. The results are presented separately for the incident beam and the secondary ions, as these two components can have significantly different angular distributions.

Figure 6 (a) and (b) show the $\left\langle l_{\text {Path }}\right\rangle$ calculated separately for the primary and secondary ions, respectively, using the $10 \mu \mathrm{m}$ diameter design when irradiated with a $150 \mathrm{MeV}$ pristine proton beam. Similarly, 6 (c) and (d) show the calculated $\left\langle l_{\text {Path }}\right\rangle$ values for a $20 \mu \mathrm{m}$ diameter $\mathrm{SV}$. The SVs are set in different positions along the Bragg curve (plotted in red) in the water phantom. The error bars plotted on figure 6 as well as all following figures represents one standard deviation calculated over ten sets of simulation data.

When considering the $\left\langle l_{\text {Path }}\right\rangle$ of incident primary protons with the detector being perfectly aligned in the phantom $\left(0^{\circ}\right.$ tilt angle $)$, the $\left\langle l_{\text {Path }}\right\rangle$ slightly decreases with increased depth in the phantom. This is due to the scattering of the beam causing an increased angle of incidence on the SV at deeper positions. At the Bragg peak the $10 \mu \mathrm{m}$ diameter SV has a value of $\sim 9 \mu \mathrm{m}$ while the $20 \mu \mathrm{m}$ diameter is $\sim 9.5 \mu \mathrm{m}$.

The results show that the value of $\left\langle l_{\text {Path }}\right\rangle$ has a strong dependence with respect to the tilt angle, with the larger diameter SV being affected less. For larger tilt angles, the $\left\langle l_{\text {Path }}\right\rangle$ of the incident beam undergoes less variation in its value. Furthermore, the secondary ion's $\left\langle l_{\text {Path }}\right\rangle$ are not seen to vary significantly for both the $10 \mu \mathrm{m}$ or $20 \mu \mathrm{m}$ designs. This effect agrees with what was predicted in figure 3 which showed that the $\left\langle l_{G e o}\right\rangle$ values converge for different tilt angles for both the designs at a cone angle $(\theta)$ of $\sim 60^{\circ}$. In particular the $\left\langle l_{\text {Path }}\right\rangle$ ranges between 
(a)

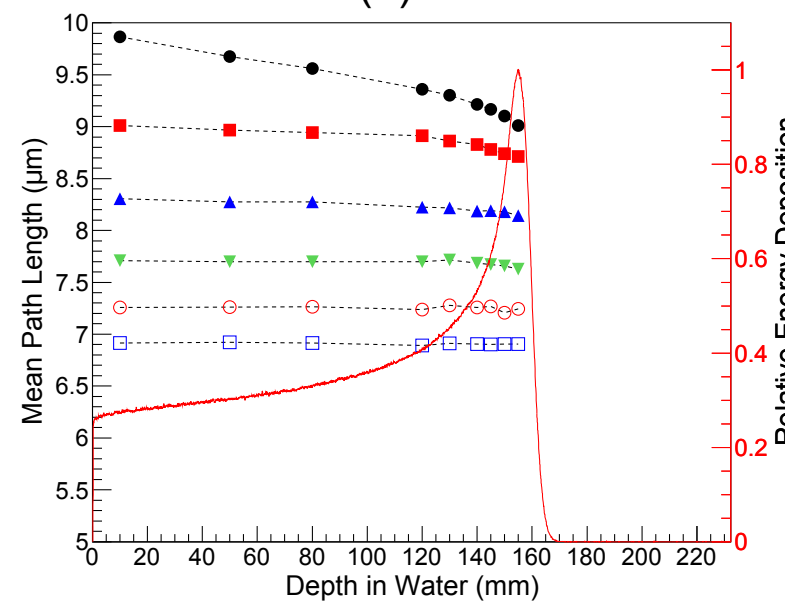

(c)

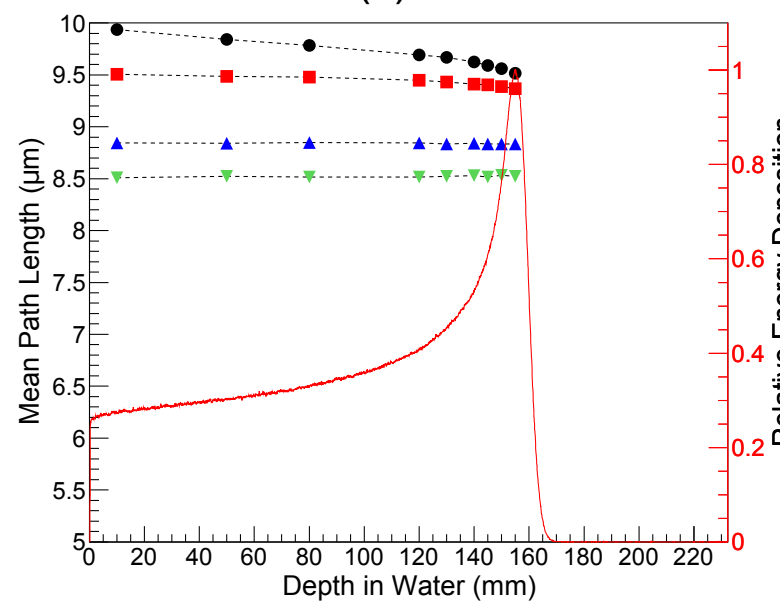

(b)

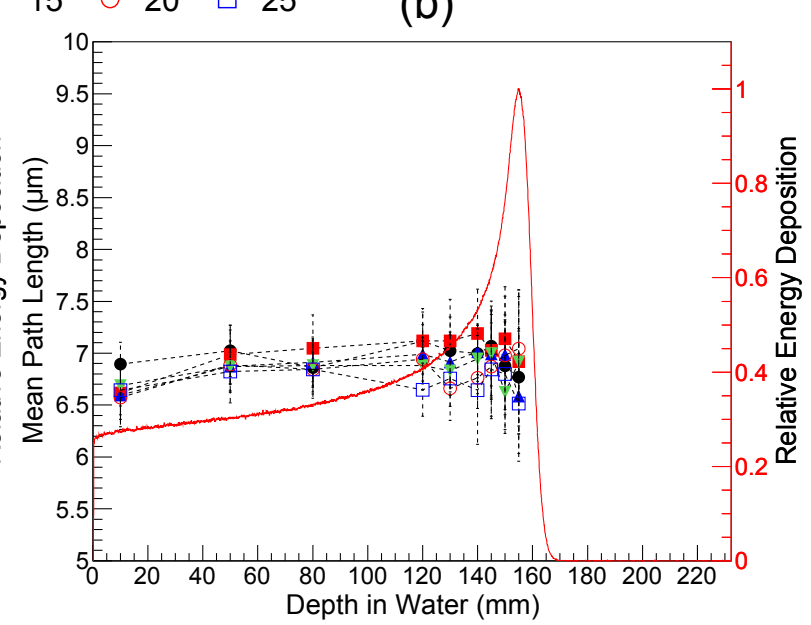

(d)

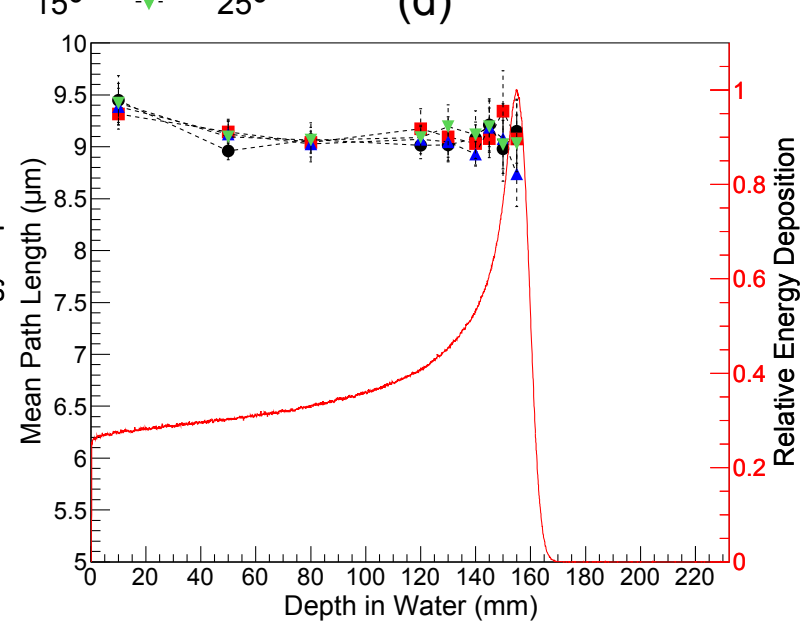

Figure 6: The $\left\langle l_{\text {Path }}\right\rangle$ calculated in a mono-energetic $150 \mathrm{MeV}$ proton beam. (a) and (b) are calculated using the $10 \mu \mathrm{m}$ diameter SV design for primary (a) and secondary ions (b), respectively. (c) and (d) also show the $\left\langle l_{\text {Path }}\right\rangle$ for primary and secondary ions, respectively, using a $20 \mu \mathrm{m}$ diameter SV instead. The microdosimeter is placed at various tilt angles with respect to the direction of the beam (reported in the legends).

$\sim 6.5$ and $7 \mu \mathrm{m}$ and $\sim 9$ and $9.5 \mu \mathrm{m}$, for the $10 \mu \mathrm{m}$ and $20 \mu \mathrm{m}$ diameter microdosimeter designs, respectively.

The $\left\langle l_{\text {Path }}\right\rangle$ of secondary ions for the $10 \mu \mathrm{m}$ design is close to the isotropic value of $6.7 \mu \mathrm{m}$, while for the $20 \mu \mathrm{m}$ design the $\left\langle l_{\text {Path }}\right\rangle$ is slightly lower than the isotropic value of $10 \mu \mathrm{m}$, with a value of $9 \mu \mathrm{m}$, which is expected for a large angular distribution based on figure 3 corresponding to a cone angle of $\sim 60^{\circ}$. At the entrance of the phantom the $\left\langle l_{\text {Path }}\right\rangle$ of the $10 \mu \mathrm{m}$ design increases slightly while the $20 \mu \mathrm{m}$ decreases from $\sim 9.4 \mu \mathrm{m}$, from figure 3 this indicates the angular distribution reaching the detector is larger at the surface of the phantom and decreases as more forwarded directed particles stay within the beam.

The $\left\langle l_{\text {Path }}\right\rangle$ calculated for 10 and $20 \mu \mathrm{m}$ diameter SV designs in a mono-energetic $290 \mathrm{MeV} / \mathrm{u}$ ${ }^{12} \mathrm{C}$ ion beam is shown in figure $7(\mathrm{a}-\mathrm{b})$ and $(\mathrm{c}-\mathrm{d})$, respectively. It can be seen that the primary ${ }^{12} \mathrm{C}$ ion beam has a noticeably larger $\left\langle l_{\text {Path }}\right\rangle$ than for the proton beam due to it having less scattering along its trajectory. 
As figure 4 shows, the fluence of secondary ions in the ${ }^{12} \mathrm{C}$ ion beam is orders of magnitude higher than for the proton beam. As observed in section 3.2, this higher fluence of fragments leads to noticeable differences between the secondary ion's $\left\langle l_{\text {Path }}\right\rangle$ of proton and ${ }^{12} \mathrm{C}$ ion beams. As discussed in [13], the secondary particles created via fragmentation in the ${ }^{12} \mathrm{C}$ ion beam tend to be more forwarded directed. Thus, they have a path length in the SV closer to the thickness of the SV, at any position along the Bragg curve. In the case of the proton beam, instead the secondary ions have a larger angular spread when reaching the SV, especially at the Bragg peak, producing a more constant $\left\langle l_{\text {Path }}\right\rangle$. This explains why the effect of the tilt angle is more pronounced in the ${ }^{12} \mathrm{C}$ ion beam case.
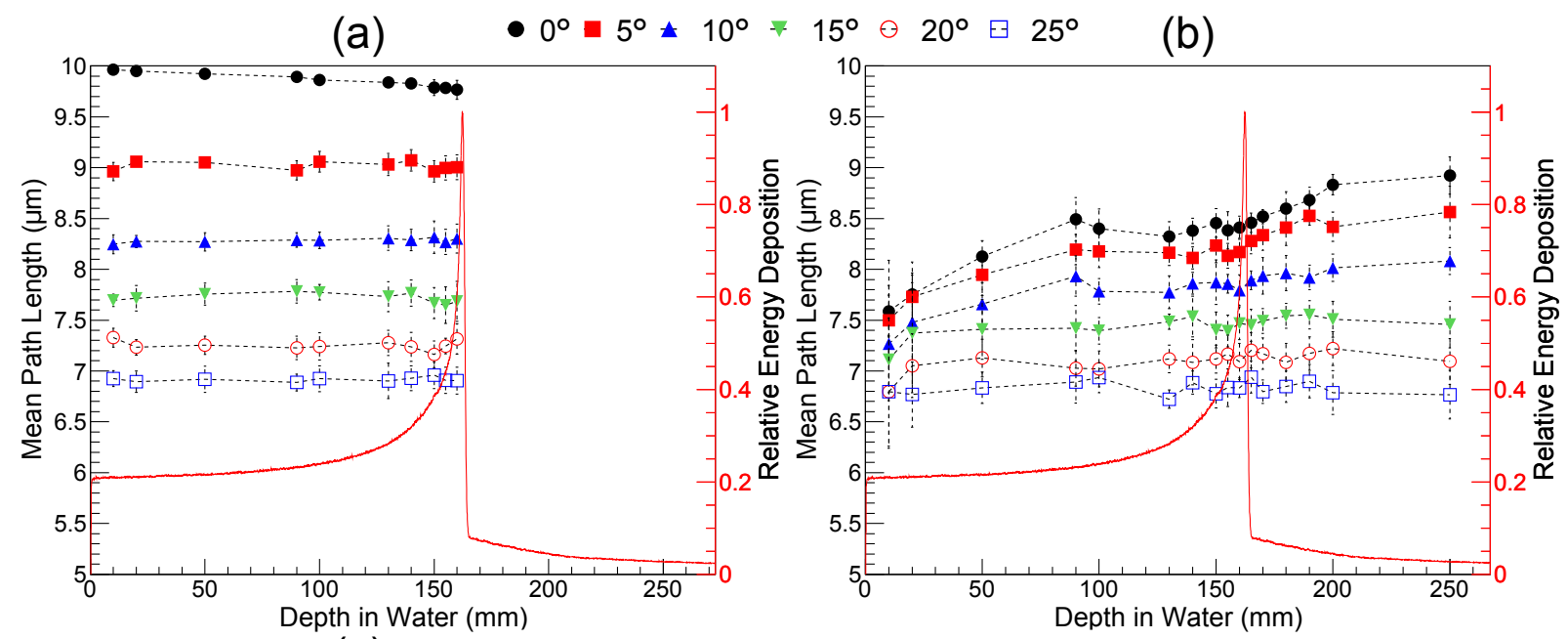

(c)

- $0^{\circ}-5^{\circ}$

$10^{\circ}$

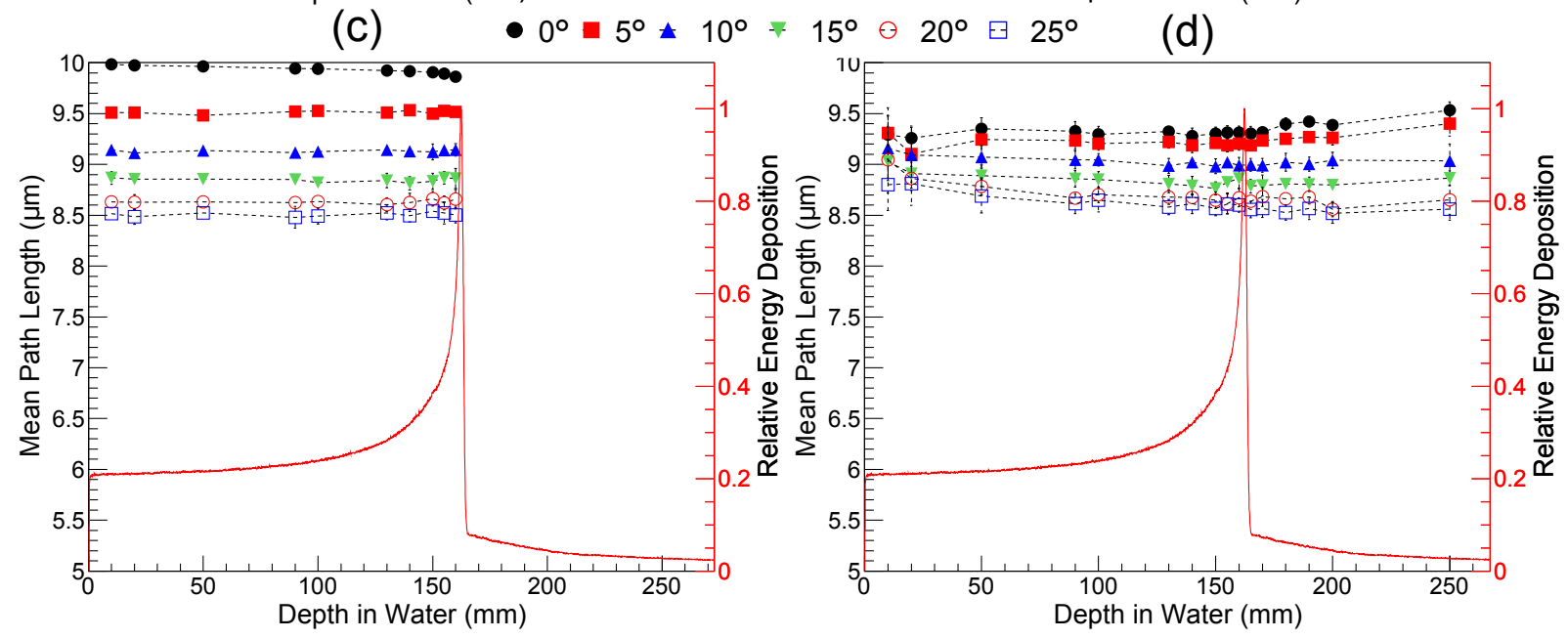

Figure 7: The $\left\langle l_{\text {Path }}\right\rangle$ calculated in a mono-energetic $290 \mathrm{MeV} / \mathrm{u}$ carbon beam. (a) and (b) are calculated using $10 \mu \mathrm{m}$ diameter SV design for primary (a) and secondary ions (b), respectively. (c) and (d) also show the $\left\langle l_{\text {Path }}\right\rangle$ for primary and secondary ions, respectively, using a $20 \mu \mathrm{m}$ diameter SV instead. The microdosimeter is placed at various tilt angles with respect to the direction of the beam (reported in the legends).

Tables 2 and 3 report the variation of the $\left\langle l_{\text {Path }}\right\rangle$ for different SV diameters placed in the monoenergetic $150 \mathrm{MeV}$ proton and $290 \mathrm{MeV} / \mathrm{u}^{12} \mathrm{C}$ ion fields. The average percentage variation, $P V$, is calculated using equation 4 , where $d$ is the number of depths in the phantom under study and $\left\langle l_{\text {Path }}\right\rangle_{0}$ is the $\left\langle l_{\text {Path }}\right\rangle$ of the SV at the first depth in the phantom. The $P V$ is calculated separately for the incident primary beam $\left(P V_{P}\right)$ and the secondary ions, $\left(P V_{S}\right)$. Equation 5 
was used to calculate the variation of the $\left\langle l_{\text {Path }}\right\rangle$ along the depth in the phantom between the primary and secondary ions $\left(P V_{P, S}\right)$. The percentage variation $P V_{t}$ was calculated for different tilt angles from the $0^{\circ}$ position between the microdosimeter and the incident beam by means of equation 6 , where $\left\langle l_{\text {Path }}\right\rangle_{i, 0}$ is the $\left\langle l_{\text {Path }}\right\rangle$ at the $i^{t h}$ depth with a tilt angle of $0^{\circ} . P V_{t}$ was also calculated along the depth of the Bragg curve. Also in this case, the $P V_{t}$ was calculated separately for the incident primary beam $\left(P V_{t, P}\right)$ and secondary ions $\left(P V_{t, S}\right)$.

$$
\begin{gathered}
\langle P V\rangle=\frac{100 \%}{d}\left(\sum_{i=0}^{d-1}\left|\frac{\left\langle l_{\text {Path }}\right\rangle_{i}-\left\langle l_{\text {Path }}\right\rangle_{0}}{\left\langle l_{\text {Path }}\right\rangle_{0}}\right|\right) \\
\left\langle P V_{P, S}\right\rangle=\frac{100 \%}{d}\left(\sum_{i=0}^{d-1}\left|\frac{\left\langle l_{\text {PathPrim }}\right\rangle_{i}-\left\langle l_{\text {PathSec }}\right\rangle_{i}}{\left\langle l_{\text {PathPrim }}\right\rangle_{i}}\right|\right) \\
\left\langle P V_{t}\right\rangle=\frac{100 \%}{d}\left(\sum_{i=0}^{d-1}\left|\frac{\left\langle l_{\text {Path }}\right\rangle_{i, t}-\left\langle l_{\text {Path }}\right\rangle_{i, 0}}{\left\langle l_{\text {Path }}\right\rangle_{i, 0}}\right|\right)
\end{gathered}
$$

\begin{tabular}{c|c|c|c|c|c|c}
\hline SV Diameter $(\mu \mathrm{m})$ & Tilt angle & $P V_{P}$ & $P V_{S}$ & $P V_{P, S}$ & $P V_{t, P}$ & $P V_{t, S}$ \\
\hline \multirow{2}{*}{10} & 0 & $4.66 \pm 0.01$ & $1.60 \pm 0.08$ & $25.71 \pm 0.39$ & $0 \pm 0$ & $0 \pm 0$ \\
& 15 & $0.23 \pm 7 \times 10^{-4}$ & $4.69 \pm 1.40$ & $11.07 \pm 0.18$ & $18.18 \pm 0.03$ & $4.98 \pm 1.63$ \\
& 25 & $0.13 \pm 4 \times 10^{-4}$ & $3.63 \pm 1.05$ & $2.28 \pm 0.05$ & $26.51 \pm 0.05$ & $5.44 \pm 1.09$ \\
\hline \multirow{2}{*}{20} & 0 & $2.27 \pm 8 \times 10^{-4}$ & $3.64 \pm 0.04$ & $6.33 \pm 0.04$ & $0 \pm 0$ & $0 \pm 0$ \\
& 15 & $0.06 \pm 6 \times 10^{-5}$ & $3.43 \pm 0.04$ & $3.01 \pm 0.02$ & $8.96 \pm 0.01$ & $1.26 \pm 0.03$ \\
& 25 & $0.12 \pm 1 \times 10^{-4}$ & $3.04 \pm 0.03$ & $7.39 \pm 0.06$ & $12.26 \pm 0.01$ & $0.77 \pm 0.01$ \\
\hline 30 & 0 & $1.25 \pm 7 \times 10^{-4}$ & $2.92 \pm 0.07$ & $3.63 \pm 0.07$ & $0 \pm 0$ & $0 \pm 0$ \\
& 15 & $0.18 \pm 2 \times 10^{-4}$ & $5.68 \pm 0.15$ & $10.52 \pm 0.15$ & $5.43 \pm 0.01$ & $1.88 \pm 0.08$ \\
& 25 & $0.16 \pm 3 \times 10^{-4}$ & $5.77 \pm 0.16$ & $12.54 \pm 0.21$ & $6.18 \pm 0.01$ & $2.25 \pm 0.06$ \\
\hline 40 & 0 & $0.87 \pm 4 \times 10^{-4}$ & $5.98 \pm 0.15$ & $10.07 \pm 0.18$ & $0 \pm 0$ & $0 \pm 0$ \\
& 15 & $0.08 \pm 1 \times 10^{-4}$ & $6.03 \pm 0.14$ & $14.47 \pm 0.32$ & $3.37 \pm 2 \times 10^{-3}$ & $1.16 \pm 0.04$ \\
& 25 & $0.17 \pm 3 \times 10^{-4}$ & $7.67 \pm 0.21$ & $16.29 \pm 0.29$ & $2.59 \pm 2 \times 10^{-3}$ & $3.30 \pm 0.07$ \\
\hline \multirow{2}{*}{50} & 0 & $0.63 \pm 3 \times 10^{-4}$ & $6.51 \pm 0.15$ & $14.13 \pm 0.20$ & $0 \pm 0$ & $0 \pm 0$ \\
& 15 & $0.14 \pm 2 \times 10^{-4}$ & $8.45 \pm 0.16$ & $17.55 \pm 0.23$ & $2.08 \pm 1 \times 10^{-3}$ & $1.05 \pm 0.03$ \\
& 25 & $0.22 \pm 3 \times 10^{-4}$ & $7.48 \pm 0.17$ & $19.55 \pm 0.29$ & $0.52 \pm 5 \times 10^{-4}$ & $3.87 \pm 0.08$ \\
\hline
\end{tabular}

Table 2: Percentage variation of the $\left\langle l_{P a t h}\right\rangle$, calculated for different SV diameters, when irradiated with a $150 \mathrm{MeV}$ proton beam. $P V_{P}$ is calculated along the Bragg curve with respect to the first depth considered in the phantom using equation 4 , for primary proton beam, $P V_{S}$ on the other hand is calculated using only secondary ions. $P V_{P, S}$ is the variance between the primary and secondary ion's $\left\langle l_{\text {Path }}\right\rangle$, averaged over all depths, calculated using equation $5 . P V_{t}$ is calculated along the Bragg curve, for different tilt angles with respect to $0^{\circ}$, for both primary $\left(P V_{t, P}\right)$ and secondary $\left(P V_{t, S}\right)$ ions, by means of equation 6 .

\section{4. $\left\langle l_{\text {Path }}\right\rangle$ in a $S O B P$ beam}

Figure $8(\mathrm{a}-\mathrm{b})$ and $(\mathrm{c}-\mathrm{d})$ show the $\left\langle l_{P a t h}\right\rangle$ for primary and secondary ions for the $10 \mu \mathrm{m}$ and 20 $\mu \mathrm{m}$ SV diameter, respectively, when irradiated with a proton beam producing a clinical SOBP. The $\left\langle l_{\text {Path }}\right\rangle$ calculated when the microdosimeter is placed in a SOBP produced by a clinical ${ }^{12} \mathrm{C}$ ion beam is shown in figure $9(\mathrm{a}-\mathrm{b})$ and $(\mathrm{c}-\mathrm{d})$, for 10 and $20 \mu \mathrm{m}$ SV diameters, respectively.

For both the proton and ${ }^{12} \mathrm{C}$ ion beams, the results are similar to the mono-energetic cases (see figures 6 and 7 ). The $20 \mu \mathrm{m}$ diameter is again preferable because the $\left\langle l_{\text {Path }}\right\rangle$ varies less along the Bragg curve and the variance between the primary and secondary $\left\langle l_{\text {Path }}\right\rangle$ is greatly reduced. 


\begin{tabular}{c|c|c|c|c|c|c}
\hline SV Diameter $(\mu \mathrm{m})$ & Tilt angle & $P V_{P}$ & $P V_{S}$ & $P V_{P, S}$ & $P V_{t, P}$ & $P V_{t, S}$ \\
\hline 10 & 0 & $1.06 \pm 3 \times 10^{-3}$ & $10.65 \pm 0.20$ & $16.49 \pm 0.20$ & $0 \pm 0$ & $0 \pm 0$ \\
& 15 & $0.57 \pm 4 \times 10^{-3}$ & $4.57 \pm 0.08$ & $4.18 \pm 0.07$ & $21.67 \pm 0.13$ & $11.31 \pm 0.10$ \\
& 25 & $0.25 \pm 2 \times 10^{-3}$ & $0.83 \pm 0.02$ & $1.30 \pm 0.02$ & $29.85 \pm 0.18$ & $18.49 \pm 0.16$ \\
\hline 20 & 0 & $0.53 \pm 4 \times 10^{-4}$ & $0.55 \pm 0.01$ & $6.30 \pm 0.02$ & $0 \pm 0$ & $0 \pm 0$ \\
& 15 & $0.24 \pm 1 \times 10^{-3}$ & $2.09 \pm 0.01$ & $0.53 \pm 0.00$ & $10.92 \pm 0.03$ & $5.33 \pm 0.02$ \\
& 25 & $0.20 \pm 8 \times 10^{-4}$ & $2.11 \pm 0.02$ & $1.71 \pm 0.02$ & $14.33 \pm 0.04$ & $7.74 \pm 0.03$ \\
\hline 30 & 0 & $0.38 \pm 4 \times 10^{-4}$ & $3.66 \pm 0.05$ & $2.30 \pm 0.01$ & $0 \pm 0$ & $0 \pm 0$ \\
& 15 & $0.17 \pm 1 \times 10^{-3}$ & $7.38 \pm 0.12$ & $2.98 \pm 0.07$ & $6.70 \pm 0.03$ & $2.58 \pm 0.02$ \\
& 25 & $0.45 \pm 3 \times 10^{-3}$ & $7.30 \pm 0.07$ & $3.98 \pm 0.05$ & $7.56 \pm 0.03$ & $2.54 \pm 0.02$ \\
\hline 40 & 0 & $0.26 \pm 2 \times 10^{-4}$ & $4.77 \pm 0.05$ & $1.56 \pm 0.02$ & $0 \pm 0$ & $0 \pm 0$ \\
& 15 & $0.24 \pm 1 \times 10^{-3}$ & $6.25 \pm 0.08$ & $4.21 \pm 0.06$ & $4.24 \pm 0.01$ & $1.12 \pm 0.01$ \\
& 25 & $0.41 \pm 3 \times 10^{-3}$ & $6.87 \pm 0.07$ & $4.44 \pm 0.05$ & $3.54 \pm 0.01$ & $0.61 \pm 0.01$ \\
\hline 50 & 0 & $0.23 \pm 1 \times 10^{-4}$ & $6.65 \pm 0.07$ & $2.13 \pm 0.03$ & $0 \pm 0$ & $0 \pm 0$ \\
& 15 & $0.14 \pm 4 \times 10^{-4}$ & $6.42 \pm 0.06$ & $4.51 \pm 0.06$ & $2.80 \pm 0.01$ & $0.67 \pm 0.01$ \\
& 25 & $0.16 \pm 8 \times 10^{-4}$ & $6.89 \pm 0.07$ & $5.16 \pm 0.05$ & $1.13 \pm 3 \times 10^{-3}$ & $1.80 \pm 0.01$ \\
\hline
\end{tabular}

Table 3: Percentage variation of the $\left\langle l_{\text {Path }}\right\rangle$, calculated for different SV diameters, when irradiated with a $290 \mathrm{MeV} / \mathrm{u}{ }^{12} \mathrm{C}$ ion beam. $P V_{P}$ is calculated along the Bragg curve with respect to the first depth considered in the phantom using equation 4 , for the incident ${ }^{12} \mathrm{C}$ ion beam, $P V_{S}$ on the other hand is calculated using only secondary ions. $P V_{P, S}$ is the variance between the primary and secondary ion's $\left\langle l_{P a t h}\right\rangle$, averaged over all depths, calculated using equation 5. $P V_{t}$ is calculated along the Bragg curve, for different tilt angles with respect to $0^{\circ}$, for both primary $\left(P V_{t, P}\right)$ and secondary $\left(P V_{t, S}\right)$ ions, by means of equation 6 .

3.4.1. Estimating the $\left\langle l_{\text {Path }}\right\rangle$ using the deposited energy Figure 10 shows the $\left\langle l_{\text {Path }}\right\rangle$ with respect to the energy deposited in the $10 \mu \mathrm{m}$ diameter silicon SV and converted to be representative of muscle. The $10 \mu \mathrm{m}$ design was used to represent an extreme case of variation between the $\left\langle l_{\text {Path }}\right\rangle$ of primary and secondary particles as well as along the depth of the Bragg curve, where using the thickness of the SV as an approximation is not appropriate, namely for proton fields. The $\left\langle l_{\text {Path }}\right\rangle$ is calculated for the primary and secondary ions, separately, with the primary ions being depicted with solid markers and secondary ions with open markers. The left plot shows the $\left\langle l_{\text {Path }}\right\rangle$ for both a mono-energetic and a SOBP proton beam and the right plot shows the same quantities for ${ }^{12} \mathrm{C}$ ion beams. For both proton and ${ }^{12} \mathrm{C}$ ion beams, it can be seen that the relationship of the $\left\langle l_{\text {Path }}\right\rangle$ with the mean peak energy deposition is valid for both a pristine beam and a SOBP which is comprised of a large range of different energy particles. This method, which allows for a quick experimental estimation of the $\left\langle l_{\text {Path }}\right\rangle$ using just the energy deposition spectra is valid not only for mono-energetic ${ }^{12} \mathrm{C}$ ion beams, but also for mono-energetic proton and SOBP proton and ${ }^{12} \mathrm{C}$ ion beams.

\section{Discussion}

The study of the $\left\langle l_{G e o}\right\rangle$ characterised the response of the different SVs of a range of different alignments with the beam and different beam divergences. The larger diameter SVs were found to show less sensitivity to alignment than smaller diameters, while the smaller diameters saw less variation in the $\left\langle l_{\text {Path }}\right\rangle$ over the complete range of cone angles $\left(0-90^{\circ}\right)$ than the larger diameters. As can been seen in [21], which measured the angular spread of the primary and secondary ions for a ${ }^{12} \mathrm{C}$ ion beam in a water phantom, the smaller $\theta$ angles mainly correspond to the incident primary beam while fragments relate to moderate sized angles. The larger $\theta$ angles are more representative of electrons and recoiled nuclei from neutrons. The larger diameters showed smaller variation for smaller cone angles of $\sim 20^{\circ}$ and less. For larger cone angles the $\left\langle l_{G e o}\right\rangle$ varied more for larger diameters with its value increasing with larger cone angles. This means that for primary beams with their smaller divergence the $\left\langle l_{P a t h}\right\rangle$ will vary less for larger 
(a) $\quad 0^{\circ}=5^{\circ} \star 10^{\circ} \forall 15^{\circ} \ominus 20^{\circ} \boxminus 25^{\circ} \quad$ (b)
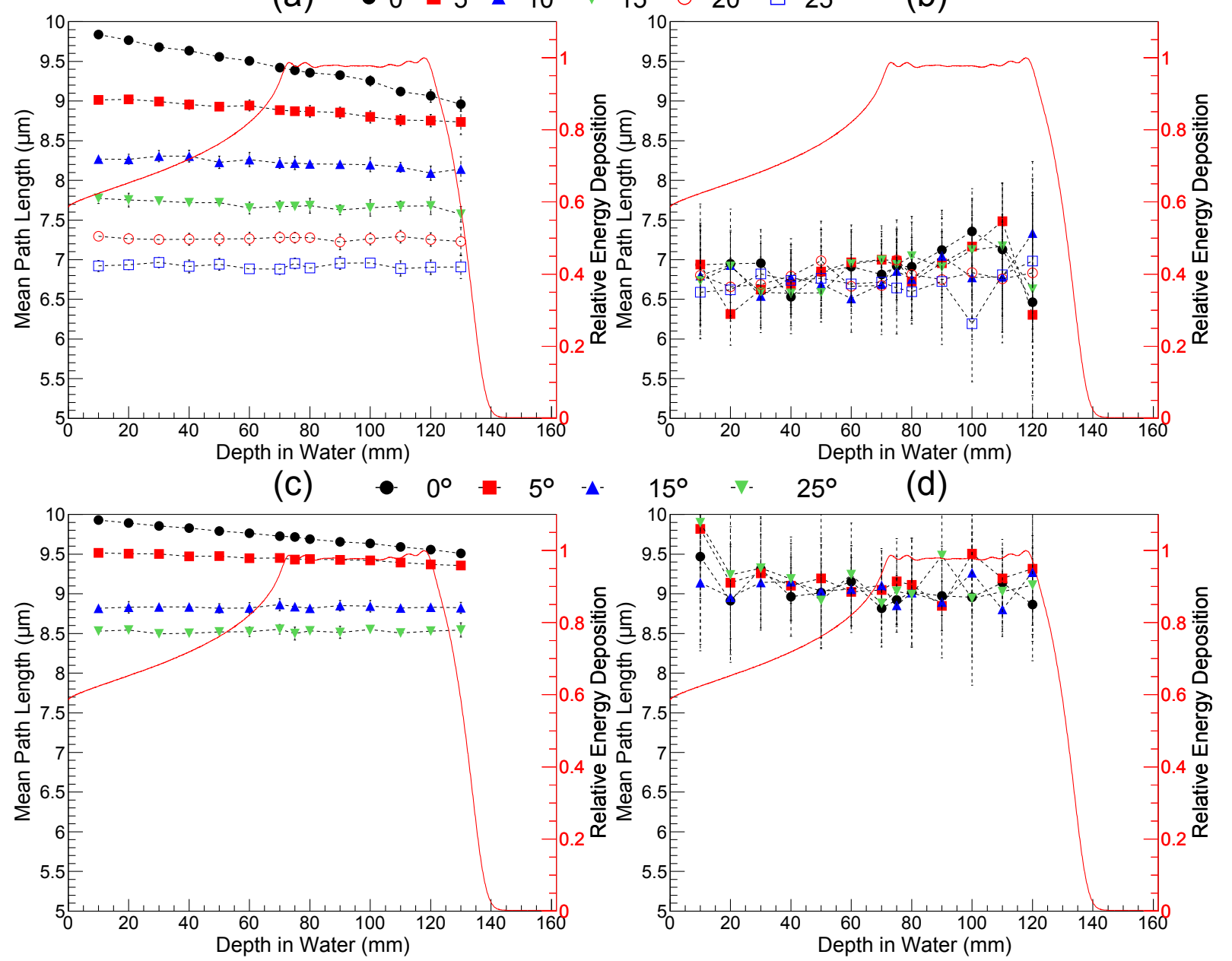

Figure 8: The $\left\langle l_{\text {Path }}\right\rangle$ when irradiated by a SOBP proton beam. (a) and (b) are calculated using $10 \mu \mathrm{m}$ diameter SV design for primary (a) and secondary ions (b), respectively. (c) and (d) also show the $\left\langle l_{\text {Path }}\right\rangle$ for primary and secondary ions, respectively, using a $20 \mu \mathrm{m}$ diameter SV instead. The microdosimeter is placed at various tilt angles with respect to the direction of the beam (reported in the legends).

diameters, however the $\left\langle l_{P a t h}\right\rangle$ of secondary particles will differ increasingly more compared to the $\left\langle l_{\text {Path }}\right\rangle$ of the primary beam as the divergence increases.

When considering the incident proton beam, the largest percentage variation observed in table 2 is with the $10 \mu \mathrm{m}$ diameter (elongation $=1$ ). This SV geometry corresponds to the least variation of the $\langle l\rangle$ when calculated by means of the Cauchy formula. The percentage variation of the primary beam, $P V_{P}$, along the depth of the Bragg curve decreases with increasing diameters for a tilt angle of $0^{\circ}$ from $4.7 \%$ to $0.6 \%$. However, as the tilt angle is increased the $P V_{P}$ is reduced for all diameters and the $20 \mu \mathrm{m}$ size has the least variance.

For secondary ions the percentage variation, $P V_{S}$, at a $0^{\circ}$ tilt angle showed the $10 \mu \mathrm{m}$ diameter to be the smallest with $2 \%$ and increases for larger diameter SVs. The reason for this can be seen in figure 3 which showed that the $\left\langle l_{\text {Path }}\right\rangle$ varied more for larger diameters when the divergences was more than $\sim 40^{\circ}$ to $90^{\circ}$, which is representative of the secondary radiation field for proton beams.

The $20 \mu \mathrm{m}$ design has the least variance between primary and secondary ions, $\left(P V_{P, S}\right)$, with 
(a)

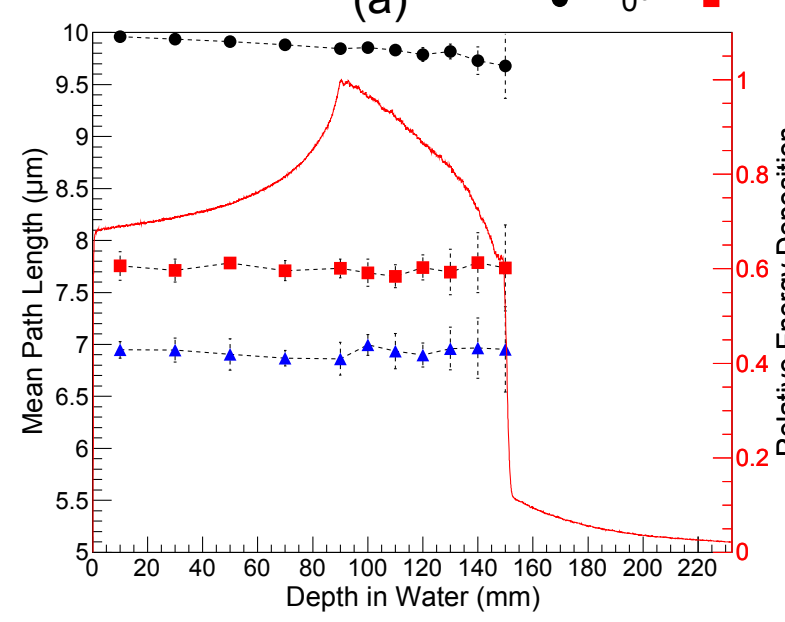

(c) $15^{\circ}$

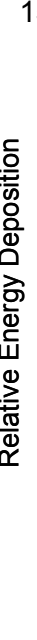

(b)

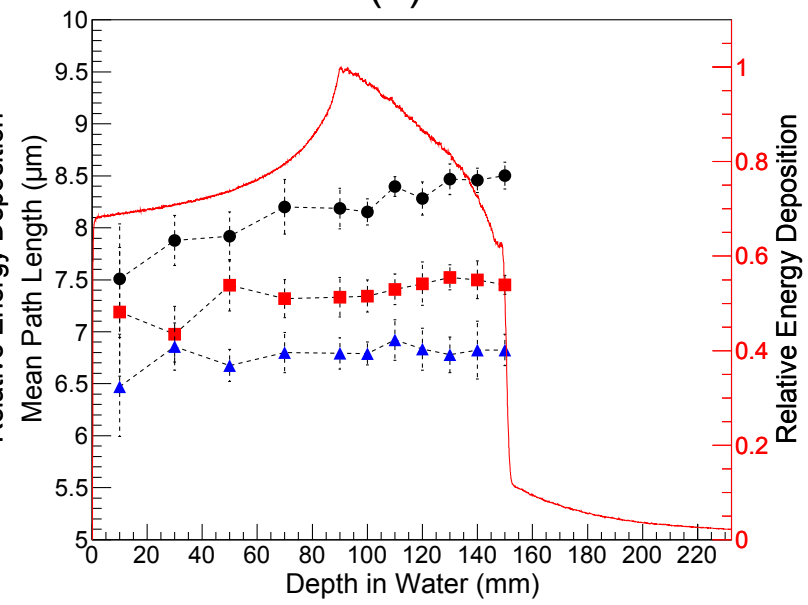

(c)

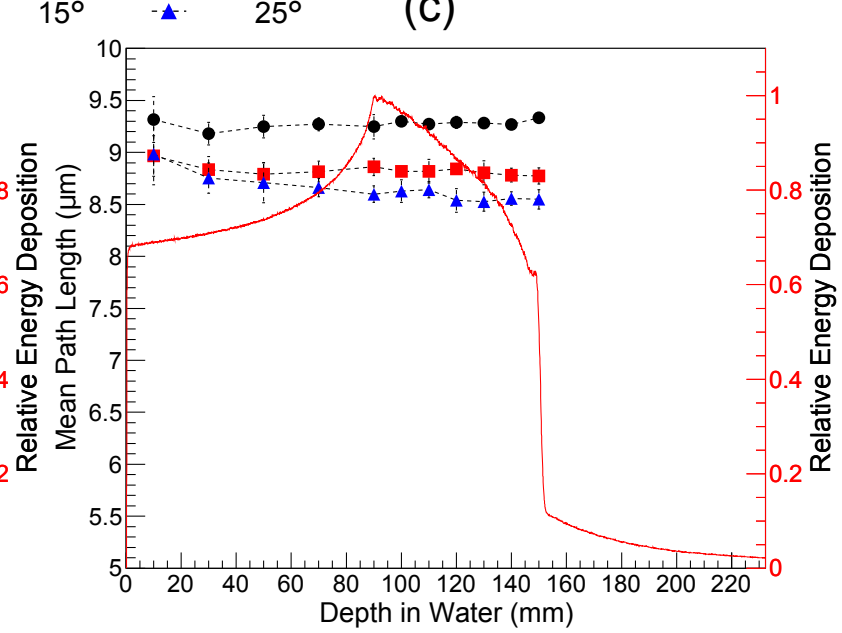

Figure 9: The $\left\langle l_{\text {Path }}\right\rangle$ when irradiated by a SOBP ${ }^{12} \mathrm{C}$ ion beam. (a) and (b) are calculated using $10 \mu \mathrm{m}$ diameter SV design for primary (a) and secondary ions (b), respectively. (c) and (d) also show the $\left\langle l_{\text {Path }}\right\rangle$ for primary and secondary ions, respectively, using a $20 \mu \mathrm{m}$ diameter $\mathrm{SV}$ instead. The microdosimeter is placed at various tilt angles with respect to the direction of the beam (reported in the legends).

the exception of extreme angles where the $10 \mu \mathrm{m}$ design varies the least, with larger diameter designs having increasingly more variance between primary and secondary ions .

When comparing the sensitivity of alignment with the $P V_{t}$ values in the last two columns it can be seen that the $10 \mu \mathrm{m}$ design is the most sensitive to alignment, particularly for the primary beam which is the most important aspect for reducing the variation. It can be seen that as the diameter of the SV increases the $P V_{t}$ for primary protons decreases slightly while for secondary ions the $P V_{t}$ is similar, but as the tilt angle increases the $P V_{t}$ becomes larger for larger diameters.

As discussed earlier, the primary proton beam and the secondary ions have similar lineal energies, with the consequence that different $\left\langle l_{\text {Path }}\right\rangle$ values cannot be applied to separate components of the microdosimetric spectrum. Thus the $\left\langle l_{P a t h}\right\rangle$ of primary and secondary ions should be similar to one another. Taking this factor into account the $20 \mu \mathrm{m}$ diameter SV, with the $\left\langle l_{\text {Cauchy }}\right\rangle$ being equal to the SV thickness, is the most appropriate design for proton therapy microdosimetric measurements. Furthermore, with the exception of downstream BP proton 


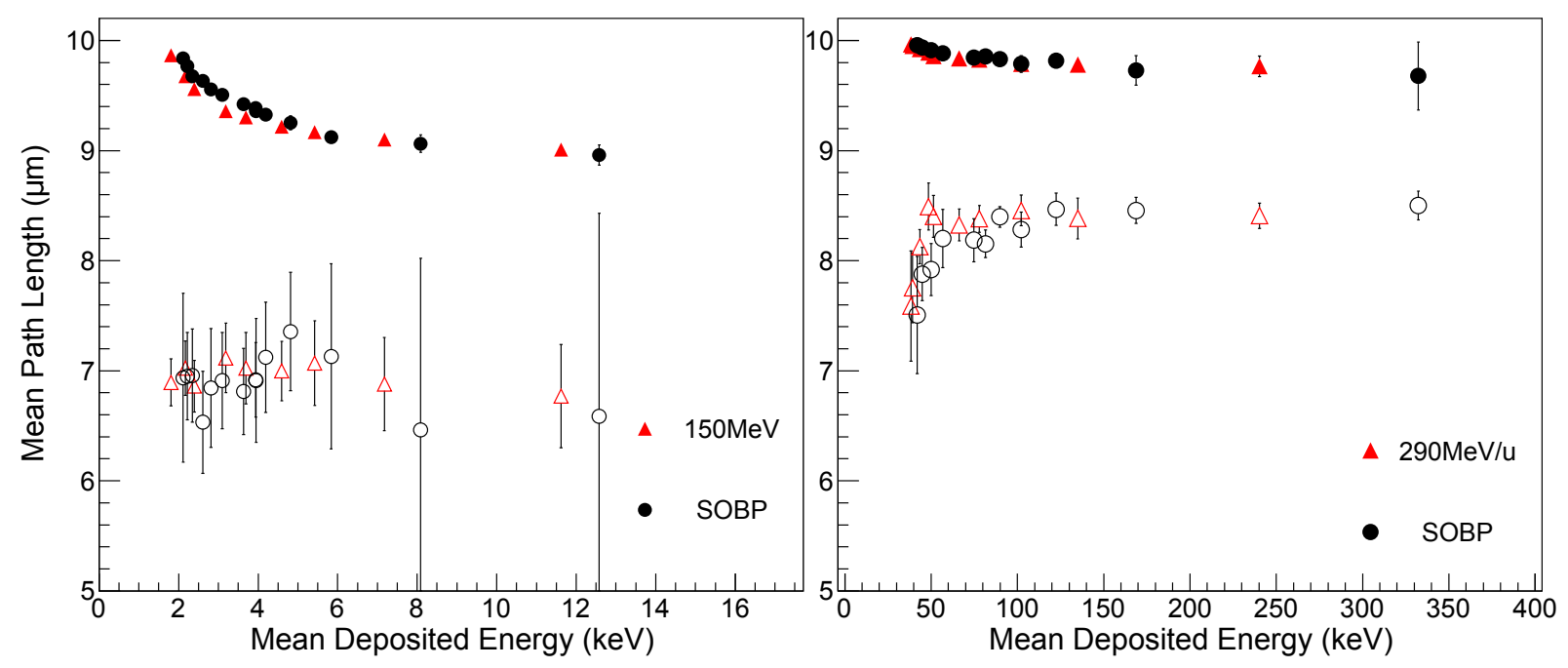

Figure 10: $\left\langle l_{\text {Path }}\right\rangle$ plotted against the mean energy deposition. Left depicts the $\left\langle l_{\text {Path }}\right\rangle$ for a $150 \mathrm{MeV}$ proton beam against a SOBP. Right depicts the $\left\langle l_{P a t h}\right\rangle$ for a $290 \mathrm{MeV} / \mathrm{u}{ }^{12} \mathrm{C}$ ion beam against a SOBP. The filled markers represent the primary beam while the open markers represent the secondary ions. Note that the energy deposited has been converted from silicon to muscle using the same methods as in [13].

measurements where the $\left\langle l_{\text {Path }}\right\rangle$ does not vary, SV designs with the $\left\langle l_{\text {Cauchy }}\right\rangle$ being less than the thickness of the detector should be avoided in proton therapy due to their large differences between their $\left\langle l_{\text {Path }}\right\rangle$ values for primary and secondary ions and larger variances with angular misalignment.

The superiority of the $20 \mu \mathrm{m}$ design with its reduced variance of the $\left\langle l_{\text {Path }}\right\rangle$ between the primary and secondary ions can be matched by the $10 \mu \mathrm{m}$ diameter design. Since the variance of the $\left\langle l_{\text {Path }}\right\rangle\left(P V_{P, S}\right)$ reduces with increasing tilt angle compared to larger designs which have increasing variance with diameter. So if the $10 \mu \mathrm{m}$ design was intentionally placed at a tilt angle of $30^{\circ}$ the variation would be insignificant, however this approach is not ideal since it diminishes the spatial resolution.

The summary of the variation in the $\left\langle l_{\text {Path }}\right\rangle$ for the ${ }^{12} \mathrm{C}$ ion beam shown in table 3 showed an increase in sensitivity to alignment by $\sim 2 \%$ compared to the proton beam for both the primary and secondary ions. This increase variation is due to the decreased angular divergence of both primary and secondary ions in ${ }^{12} \mathrm{C}$ ion beams.

When considering the incident ${ }^{12} \mathrm{C}$ ion beam only, the $P V_{P}$ is less than $1.1 \%$ for all diameter sizes because of the small divergence of the ${ }^{12} \mathrm{C}$ ions, with $P V_{P}$ decreasing slightly with increasing diameters. The $P V_{S}$ for secondary ions has similar values as proton beams, a noticeable exception is for $10 \mu \mathrm{m}$ which has a value of $\sim 11 \%$ compared to $\sim 2 \%$, this is due to the complex radiation field in ${ }^{12} \mathrm{C}$ ion beams as the angular distribution and dominance of particles changes with depth.

When considering the variation between the primary and secondary ion's $\left\langle l_{P a t h}\right\rangle\left(P V_{P, S}\right)$, a SV diameter of $20-30 \mu \mathrm{m}$ has the smallest variance of $\left\langle l_{P a t h}\right\rangle$ and in general the $P V_{P, S}$ is smaller in ${ }^{12} \mathrm{C}$ due to the dominance of forward directed fragments which have angular distributions much closer to the primary beam compared to neutron events. The diameter sizes of 20-30 $\mu \mathrm{m}$ correspond closely to the real world Mushroom and Bridge devices.

The sensitivity of the $\left\langle l_{\text {Path }}\right\rangle$ on the detector alignment showed that the values of $P V_{t, P}$ are similar to what was seen for the proton beam, with larger diameters having a decreased variation. The values of $P V_{t, P}$ for ${ }^{12} \mathrm{C}$ ions is slightly higher than for proton, the reason for this can be 
seen in figure 3 which showed that the largest variation in the $\left\langle l_{P a t h}\right\rangle$ (or $\left\langle l_{G e o}\right\rangle$, as in the figure) for smaller beam divergence. For $P V_{t, S}$ it can be seen that the variation is again similar to what was seen with proton except that for smaller diameters the $P V_{t, S}$ is greater while for larger diameters the $P V_{t, S}$ is smaller.

Thanks to the ability to distinguish between the primary and secondary ions in ${ }^{12} \mathrm{C}$ ion therapy, the choice of SV diameter is less important compared with proton beams and allows for a greater flexibility in the microdosimeter design. For instance, if the alignment of the detector with the incident beam is difficult, then a SV design with a larger diameter will offer an advantage due to the decreased variation in the $\left\langle l_{\text {Path }}\right\rangle$. Instead, for proton beams, the 20 $\mu \mathrm{m}$ diameter $\mathrm{SV}$ is preferable as both primary and secondary particles have closer mean path lengths.

For SOBP radiation fields the results did not differ significantly from mono-energetic beams. The $\left\langle l_{\text {Path }}\right\rangle$ has a more consistent value with a $20 \mu \mathrm{m}$ SV diameter compared to the $10 \mu \mathrm{m}$ $\mathrm{SV}$, however, as mentioned before, the choice of diameter is not as crucial in this case as the contribution to the microdosimetric spectra can be distinguished and applied to different regions of the spectra, as shown in figure 5, however for ease and simplicity this approach is not preferable.

\section{Conclusions}

The aim of this work was to identify the best diameter for a $10 \mu \mathrm{m}$ thick silicon microdosimeter $\mathrm{SV}$ to reduce the variation of the $\left\langle l_{\text {Path }}\right\rangle$, when considering different positions along the Bragg curve, alignments with the direction of the incident beam and different particles of the radiation field produced by clinical proton and ${ }^{12} \mathrm{C}$ ion beams. By reducing the variation in the $\left\langle l_{\text {Path }}\right\rangle$ allows for the energy deposition to be converted to lineal energy more accurately and easily. This is important to accurately determine the microdosimetric spectra and to estimate the RBE.

It was found that for proton beams that the most appropriate microdosimeter design has SVs with $20 \mu \mathrm{m}$ diameter. The thickness of the SV, which is $10 \mu \mathrm{m}$, is equal to the $\left\langle l_{\text {Cauchy }}\right\rangle$ of the SV calculated by means of the Cauchy formula. For ${ }^{12} \mathrm{C}$ ion beams the design of the SV was found to be less important than for protons. This is because the fragments produced have angular distributions closer to the primary beam than neutron events and if necessary the components of the microdosimetric spectra deriving from the incident beam and the secondary radiation field can be easily distinguished and use separate $\left\langle l_{\text {Path }}\right\rangle$ values. The $20 \mu \mathrm{m}$ diameter is appropriate for both pristine and SOBP proton and carbon ions.

This study shows that intentionally introducing a small misalignment may reduce the variation of the $\left\langle l_{\text {Path }}\right\rangle$, the angle required being reduced with larger diameter SV designs, with an angle of $\sim 10^{\circ}$ required for the $20 \mu \mathrm{m}$ design in a proton beam. This method has the largest applicability for proton beams where the lineal energies of the primary and secondary ions have similar lineal energies. Nevertheless the feasibility of this method has to be evaluated experimentally.

Proton beams were characterised with lower values of $\left\langle l_{\text {Path }}\right\rangle$ for both primary and secondary ions due to the larger associated scatter of the primary beam and dominance of neutrons in the generation of secondary ions. When placed in a mono-energetic proton beam the $20 \mu \mathrm{m}$ diameter design, with a $\left\langle l_{\text {Cauchy }}\right\rangle$ of $10 \mu \mathrm{m}$ the $\left\langle l_{\text {Path }}\right\rangle$ varied from $9.9 \mu \mathrm{m}$ at the entrance of the phantom to $9.5 \mu \mathrm{m}$ at the $\mathrm{BP}$ and for the ${ }^{12} \mathrm{C}$ ion beam with a change from $10 \mu \mathrm{m}$ to $9.8 \mu \mathrm{m}$. While for the $10 \mu \mathrm{m}$ diameter design with a $\left\langle l_{\text {Cauchy }}\right\rangle$ of $6.67 \mu \mathrm{m}$ finished with $\left\langle l_{\text {Path }}\right\rangle$ values of $9.0 \mu \mathrm{m}$ and $9.8 \mu \mathrm{m}$ at the BP for the proton and ${ }^{12} \mathrm{C}$ ion beams, respectively.

This work demonstrated that there is a unique relationship between the $\left\langle l_{\text {Path }}\right\rangle$ in the SV and the mean energy deposited, not only for pristine proton and ${ }^{12} \mathrm{C}$ ion beams but also for clinical SOBPs. This is particularly convenient for experimental use of the microdosimeters and allows 
for quick and accurate determination of the $\left\langle l_{\text {Path }}\right\rangle$ using the spectra of the deposited energies.

\section{Acknowledgement}

We would like to thank the Australian National Computational Infrastructure (NCI) for providing computing time on the Raijin cluster.

We would like to thank the University of Wollongong Information Technology Services (ITS) for computing time on the UOW High Performance Computing Cluster.

This research is supported by an Australian Government Research Training Program (RTP) Scholarship.

This project is funded by the Australian Research Council Discover Project grant DP170102273.

\section{References}

[1] Y. Kase, T. Kannai, Y. Matsumotot, Y. Furusawa, H. Okamato, T. Asaba, M. Sakama, and H. Shinoda, "Mirodosimetric measurements and estimation of human cell survival for heavy-ion beams," Radiation Research Society, vol. 166, pp. 629-638, 2006.

[2] Y. Kase, W. Yamashita, N. Matsufuji, K. Takada, T. Sakae, Y. Furusawa, H. Yamashita, and S. Murayama, "Microdosimetric calculation of relative biological effectiveness for design of therapeutic proton beams," Journal of Radiation Research, vol. 54, no. 3, p. 485, 2013.

[3] R. B. Hawkins, "A statistical theory of cell killing by radiation of varying linear energy transfer," Radiation Research, vol. 140, pp. 366-374, 1994.

[4] R. B. Hawkins, "A microdosimetric-kinetic model for the effect of non-poisson distribution of lethal lesions on the variation of rbe with let," Radiation Research, vol. 160, pp. 61-69, 2003.

[5] L. T. Tran, L. Chartier, D. Bolst, D. A. Prokopovich, S. Guatelli, M. Nancarrow, M. I. Reinhard, M. Petasecca, M. L. F. Lerch, V. L. Pereverlaylo, N. Matsufuji, D. Hinde, M. Dasgupta, A. Stuchbery, M. Jackson, and A. B. Rosenfeld, "3D silicon microdosimetry and RBE study using ${ }^{12} C$ ion of different energies," IEEE Transactions on Nuclear Science, vol. 62, pp. 3027-3033, Dec 2015.

[6] L. T. Tran, L. Chartier, D. A. Prokopovich, D. Bolst, M. Povoli, A. Summanwar, A. Kok, A. Pogossov, M. Petasecca, S. Guatelli, M. I. Reinhard, M. Lerch, M. Nancarrow, N. Matsufuji, M. Jackson, and A. B. Rosenfeld, "Thin silicon microdosimeter utilizing 3-d mems fabrication technology: Charge collection study and its application in mixed radiation fields," IEEE Transactions on Nuclear Science, vol. 65, pp. 467-472, Jan 2018.

[7] L. T. Tran, L. Chartier, D. Bolst, A. Pogossov, S. Guatelli, M. Petasecca, M. L. F. Lerch, D. A. Prokopovich, M. I. Reinhard, B. Clasie, N. Depauw, H. Kooy, J. B. Flanz, A. McNamara, H. Paganetti, C. Beltran, K. Furutani, V. L. Perevertaylo, M. Jackson, and A. B. Rosenfeld, "Characterization of proton pencil beam scanning and passive beam using a high spatial resolution solidstate microdosimeter," Medical Physics, vol. 44, no. 11, pp. 6085-6095.

[8] L. Chartier, L. T. Tran, D. Bolst, S. Guatelli, A. Pogossov, D. A. Prokopovich, M. I. Reinhard, V. Perevertaylo, S. Anderson, C. Beltran, N. Matsufuji, M. Jackson, and A. B. Rosenfeld, "Microdosimetric applications in proton and heavy ion therapy using silicon microdosimeters," Radiation Protection Dosimetry, pp. 1-7, 2017.

[9] L. T. Tran, D. Bolst, S. Guatelli, A. Pogossov, M. Petasecca, M. L. F. Lerch, L. Chartier, D. A. Prokopovich, M. I. Reinhard, M. Povoli, A. Kok, V. L. Perevertaylo, N. Matsufuji, T. Kanai, M. Jackson, and A. B. Rosenfeld, "The relative biological effectiveness for carbon, nitrogen, and oxygen ion beams using passive and scanning techniques evaluated with fully 3d silicon microdosimeters," Medical Physics, vol. 45, no. 5, pp. 2299-2308.

[10] H. H. Rossi and M. Zaider, Microdosimetry and its applications. Springer, 1996.

[11] A. Cauchy, "Mémoire sur la rectfication des courbes et la quadrature des surfaces courbes," Oeuvres Complétes, vol. 2, 1908.

[12] A. M. Kellerer, "Chord length distributions and related quantities for spheroids," Radiation Research, vol. 98, pp. 425-437, 1984.

[13] D. Bolst, S. Guatelli, L. Tran, L. Chartier, M. Lerch, N. Matsufuji, and A. Rosenfeld, "Correction factors to convert microdosimetry measurements in silicon to tissue in $12 \mathrm{C}$ ion therapy," Physics in Medicine and Biology, Feb 2017.

[14] J. Allison et al., "Geant4 developments and applications," IEEE Transactions on Nuclear Science, vol. 53, pp. 270-278, Feb 2006. 
[15] S. Agostinelli et al., "Geant4 - a simulation toolkit," Nuclear Instruments and Methods in Physics Research Section A: Accelerators, Spectrometers, Detectors and Associated Equipment, vol. 506, no. 3, pp. 250 303, 2003.

[16] J. Allison et al., "Recent developments in Geant4," Nuclear Instruments and Methods in Physics Research Section A: Accelerators, Spectrometers, Detectors and Associated Equipment, vol. 835, pp. 186 - 225, 2016.

[17] D. Bolst, G. A. Cirrone, G. Cuttone, G. Folger, S. Incerti, V. Ivanchenko, T. Koi, D. Mancusi, L. Pandola, F. Romano, A. B. Rosenfeld, and S. Guatelli, "Validation of Geant4 fragmentation for heavy ion therapy," Nuclear Instruments and Methods in Physics Research Section A: Accelerators, Spectrometers, Detectors and Associated Equipment, vol. 869, pp. 68 - 75, 2017.

[18] S. Guatelli, M. I. Reinhard, B. Mascialino, D. A. Prokopovich, A. S. Dzurak, M. Zaider, and A. B. Rosenfeld, "Tissue equivalence correction in silicon microdosimetry for protons characteristic of the leo space environment," IEEE Transaction on Nuclear Science, vol. 55, pp. 3407-3413, 2008.

[19] P. D. Bradley, A. B. Rosenfeld, and M. Zaider, "Solid state microdosimetry," Nuclear Instruments and Methods in Physics Research B, vol. 184, pp. 135-157, 2001.

[20] "ICRU Report 36-Microdosimetry," International Commission on Radiation Units and Measurements, 1970.

[21] N. Matsufuji, M. Komori, H. Sasaki, K. Akiu, M. Ogawa, A. Fukumura, E. Urakabe, T. Inaniwa, T. Nishio, T. Kohno, and T. Kanai, "Spatial fragment distribution from a therapeutic pencil-like carbon beam in water," Physics in Medicine and Biology, vol. 50, no. 14, p. 3393, 2005. 